ARTICLE

Received 22 Aug 2016 | Accepted 17 Mar 2017 | Published 11 May 2017

DOI: $10.1038 /$ ncomms15295

OPEN

\title{
Tau association with synaptic vesicles causes presynaptic dysfunction
}

Lujia Zhou ${ }^{1,2, \star}$, Joseph Mclnnes ${ }^{1,2, \star}$, Keimpe Wierda ${ }^{1,2}$, Matthew Holt ${ }^{1,2}$, Abigail G. Herrmann ${ }^{3}$, Rosemary J. Jackson ${ }^{3}$, Yu-Chun Wang ${ }^{1,2}$, Jef Swerts ${ }^{1,2}$, Jelle Beyens ${ }^{1,2}$, Katarzyna Miskiewicz ${ }^{1,2}$, Sven Vilain ${ }^{1,2}$, Ilse Dewachter ${ }^{4,5}$, Diederik Moechars ${ }^{6}$, Bart De Strooper ${ }^{1,2}$, Tara L. Spires-Jones ${ }^{3}$, Joris De Wit ${ }^{1,2}$ \& Patrik Verstreken ${ }^{1,2}$

Tau is implicated in more than 20 neurodegenerative diseases, including Alzheimer's disease. Under pathological conditions, Tau dissociates from axonal microtubules and missorts to preand postsynaptic terminals. Patients suffer from early synaptic dysfunction prior to Tau aggregate formation, but the underlying mechanism is unclear. Here we show that pathogenic Tau binds to synaptic vesicles via its $\mathrm{N}$-terminal domain and interferes with presynaptic functions, including synaptic vesicle mobility and release rate, lowering neurotransmission in fly and rat neurons. Pathological Tau mutants lacking the vesicle binding domain still localize to the presynaptic compartment but do not impair synaptic function in fly neurons. Moreover, an exogenously applied membrane-permeable peptide that competes for Tau-vesicle binding suppresses Tau-induced synaptic toxicity in rat neurons. Our work uncovers a presynaptic role of Tau that may be part of the early pathology in various Tauopathies and could be exploited therapeutically.

\footnotetext{
${ }^{1}$ VIB-KU Leuven Center for Brain \& Disease Research, Leuven 3000, Belgium. ${ }^{2}$ KU Leuven, Department of Neurosciences, Leuven Research Institute for Neuroscience and Disease (LIND), Leuven 3000, Belgium. ${ }^{3}$ University of Edinburgh, Centre for Cognitive and Neural Systems, Center for Dementia Prevention and Euan MacDonald Centre, Edinburgh EH8 9JZ, UK. ${ }^{4}$ Catholic University of Louvain, Alzheimer Dementia Group, Institute of Neuroscience, Brussels 1200, Belgium. ${ }^{5}$ University of Hasselt, Biomedical Research Institute, Hasselt 3500, Belgium. ${ }^{6}$ A Division of Janssen Pharmaceutica NV, Neuroscience Department, Janssen Research and Development, Beerse 2340, Belgium. * These authors contributed equally to this work. Correspondence and requests for materials should be addressed to P.V. (email: patrik.verstreken@cme.vib-kuleuven.be).
} 
A ccumulation of abnormal Tau in human brain has been implicated in major neurodegenerative diseases termed Tauopathies, including Alzheimer's disease (AD), Parkinson disease (PD), and Frontotemporal dementia with Parkinsonsim-17 (FTDP-17) (reviewed in refs 1-3). More than 40 mutations in the Tau-encoding MAPT locus have been identified as a direct cause for FTDP-17, and slightly higher expression of Tau driven by the Tau $\mathrm{H} 1$ haplotype increases risk for $\mathrm{AD}$ and $\mathrm{PD}^{4-6}$. In addition, several groups have shown that loss of Tau prevents $\mathrm{A} \beta$ and other excitotoxin-induced neuronal toxicity and cognitive deficits in mouse models of $\mathrm{AD}$ and other neurodegenerative diseases, suggesting that Tau is a direct mediator of neurodegenerative disease $\mathrm{s}^{7-12}$.

Synaptic dysfunction is thought to be an early pathological manifestation in $\mathrm{AD}$ and other Tauopathies ${ }^{13-16}$. Recent studies suggest that soluble Tau, rather than the neurofibrillary tangleassociated aggregated form, is the main toxic element that is able to induce early synaptic deficits preceding synapse and neuronal loss ${ }^{17-24}$. However, pathogenic mechanisms of non-aggregated Tau in neurons remain enigmatic.

Tau is mainly expressed in neurons and is highly concentrated in the axon, where it associates with microtubules. However, under disease conditions, Tau detaches from microtubules as abnormal phosphorylation or FTDP-17 pathogenic mutations lower its binding affinity for microtubules ${ }^{3,25}$. Pathological Tau has been detected to colocalize with both pre- and post-synaptic markers in the staining of isolated synaptosomes from AD patient brains $^{26-29}$. Immunohistological analyses of transgenic mouse brains made similar observations, that FTDP-17 pathogenic mutations drive mislocalization of Tau to both presynaptic terminals and dendritic spines ${ }^{30,31}$. These data together implicate that Tau missorts to both pre- and post- synapses under pathological conditions. Several studies have suggested a pathophysiological role of Tau at dendritic spines in affecting the trafficking of postsynaptic receptors ${ }^{21,32}$. In contrast, the role for mis-localized Tau at presynaptic terminals remains unclear.

In this study, we show that FTDP-17 mutant Tau mislocalizes to presynaptic terminals in fly neurons, where it binds synaptic vesicles to elicit presynaptic dysfunction. The underlying mechanism involves a dual action of Tau in binding synaptic vesicles and simultaneously promoting presynaptic actin polymerization to crosslink vesicles and restrict their mobilization. Pathogenic Tau thus reduces synaptic transmission during prolonged neuronal activity in fly neurons, and similar defects are observed in rat neurons. Disruption of the interaction between Tau and synaptic vesicles is sufficient to rescue these defects, suggesting that the mode-of-action we identified is a key mechanism for Tau-induced presynaptic pathology.

\section{Results}

Pathogenic Tau binds to vesicles at presynaptic terminals. Pathological Tau was previously detected at both pre- and postsynaptic compartments in isolated synaptosomes from $\mathrm{AD}$ patient brains ${ }^{26,28,29}$. We applied array tomography to determine the localization of pathological Tau in situ in the brain of $\mathrm{AD}$ patients. Ultrathin $(70 \mathrm{~nm})$ brain sections were prepared and labelled with antibodies against pathological phospho-Tau (AT8) and the presynaptic marker protein Synapsin. Our data shows that pathological phospho-Tau is present at presynaptic terminals of $\mathrm{AD}$ patient brains but is absent from the presynaptic terminals of healthy controls (Supplementary Fig. 1). Our data, together with previous studies, argues for the relevance of exploring the role of presynaptic Tau in eliciting synaptic pathology.

Given that the mechanisms of presynaptic function are well conserved across species ${ }^{33,34}$, we resorted to Drosophila larval neuromuscular junctions (NMJs) to study the presynaptic role of Tau. Presynaptic terminals at the NMJ are large, allowing for imaging, and they can be genetically separated from the post-synaptic muscle. We inserted expression constructs of human wild type or FTDP-17 pathogenic mutant Tau (R406W, V337M and P301L, 0N4R isoform) at identical genomic loci in the fly genome, resulting in very similar expression levels (Supplementary Fig. 2a,b). We compared Tau expression to previously published lines ${ }^{35-38}$ and find our new constructs result in about four times lower expression levels (Supplementary Fig. 2c,d). Furthermore, the expression level of human Tau in fly brains is in a similar range of the level of Tau in human brain samples (Supplementary Fig. 2e,f). Our new lines are thus optimized to express Tau at a low level to recapitulate Tau expression in human brains.

We then assessed Tau localization in fly neurons. In motor neurons, both wild type and pathogenic mutant Tau are localized to axons. However, compared to wild type Tau, the pathogenic mutants show increased localization to presynaptic terminals labelled by the synaptic vesicle marker CSP (Fig. 1a,b). The increased presynaptic localization of the pathogenic mutant Tau is likely attributed to their lower affinity for binding microtubules, as previously reported ${ }^{3,25}$ and parallels our observations based on array tomography in human patient brains, where we also observed pathological Tau at presynaptic terminals (Supplementary Fig. 1).

We next assessed the sub-boutonic localization and distribution of synaptic-localized Tau using super-resolution structured illumination microscopy. We find that pathogenic Tau at presynaptic boutons is distributed in a 'doughnut-like' pattern that closely resembles that of synaptic vesicles marked by antiCSP labelling (Fig. 1c). We therefore questioned whether Tau associates with synaptic vesicles and hereto performed a vesicle depletion assay using a temperature sensitive Dynamin mutant $\left(s h i^{t s 1}\right)$ that blocks endocytosis at restrictive temperature ${ }^{39}$. When stimulated with $\mathrm{KCl}$, synaptic vesicles fuse with the membrane but are not retrieved by endocytosis, as shown by the re-localization of the synaptic vesicle marker CSP to the plasma membrane (Fig. 1d). Similar to CSP, Tau relocates to the plasma membrane (Fig. 1d), suggesting that mislocalized pathogenic Tau at presynaptic terminals associates with synaptic vesicles in vivo.

To analyse the vesicle-binding properties of Tau, we prepared ultrapure synaptic vesicles by controlled-pore glass chromatography from rat brain (Supplementary Fig. 3a-e) and incubated the vesicles with purified recombinant human Tau (Supplementary Fig. 3f-h). Recombinant Tau co-sediments with synaptic vesicles in a vesicle sedimentation assay (Fig. 1e). Synaptic vesicles also co-immunoprecipitate using recombinant Tau as bait, evidenced by the recovery of synaptic vesicle marker proteins (Fig. 1f). Finally, electron microscopy of recombinant Tau incubated with isolated synaptic vesicles in vitro shows the protein binds to vesicle membranes (Fig. 1g). Note that both wild type and pathogenic mutant Tau are able to bind to synaptic vesicles in vitro (Fig. 1e,f); however, only mutant Tau is prominently present at presynaptic terminals, allowing it to bind to synaptic vesicles in vivo (Fig. 1a,b).

To determine the mode of vesicle binding we generated and purified Tau-deletion proteins and assessed if they could coimmunoprecipitate synaptic vesicles. Only truncation of the N-terminus $\left(\mathrm{aa}_{1-112}\right)$ of Tau, but not the truncation of any other domain, strongly impedes with the binding of Tau to synaptic vesicles (Fig. $1 \mathrm{~h}-\mathrm{j}$ ). In agreement, the Tau N-terminal fragment alone co-immunoprecipitates vesicles (Supplementary Fig. 3i). Therefore, the N-terminus of Tau is necessary and sufficient for synaptic vesicle binding. 
a Confocal imaging of Tau labeling at NMJs
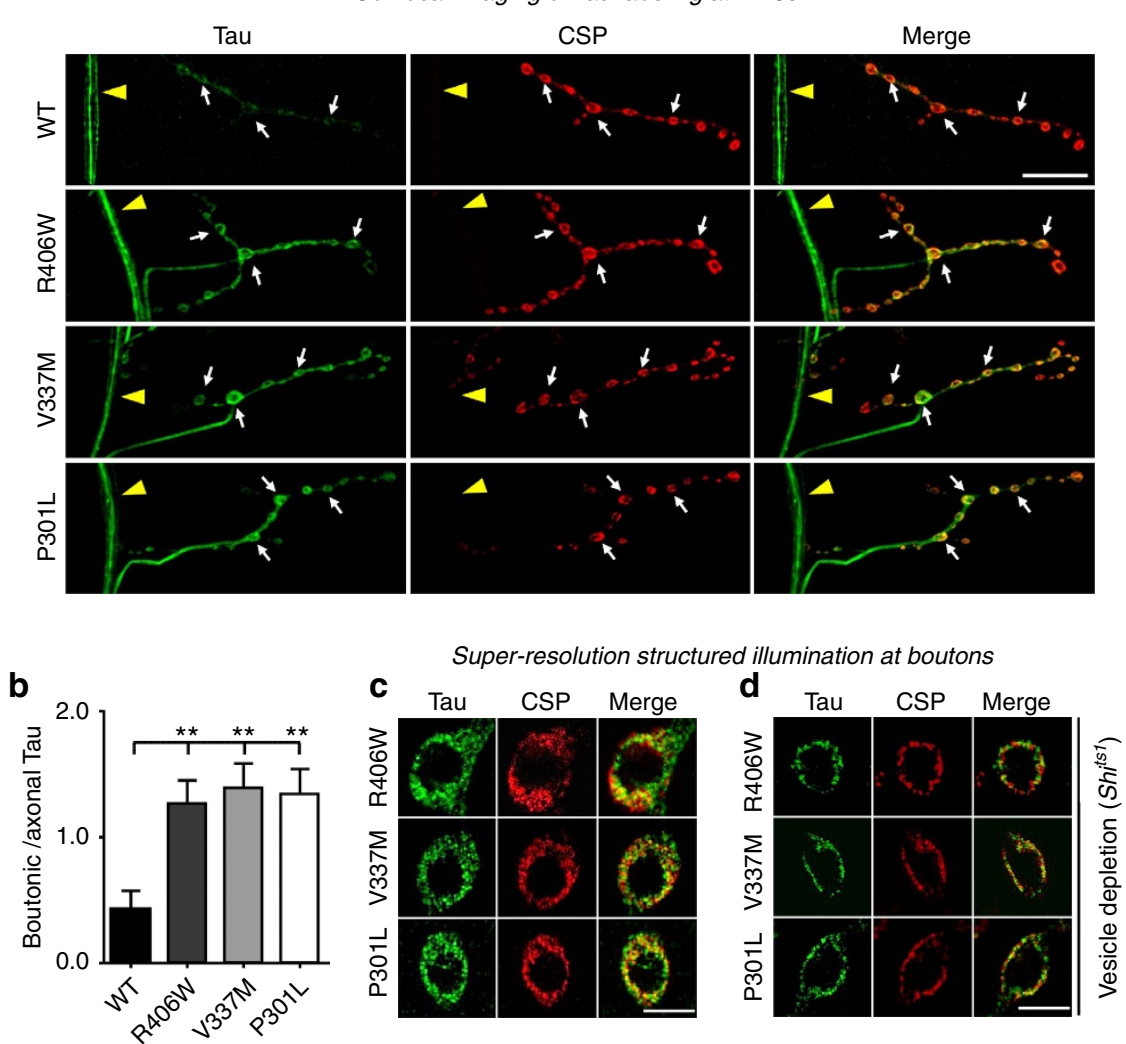

In vitro binding of Tau to isolated synaptic vesicles
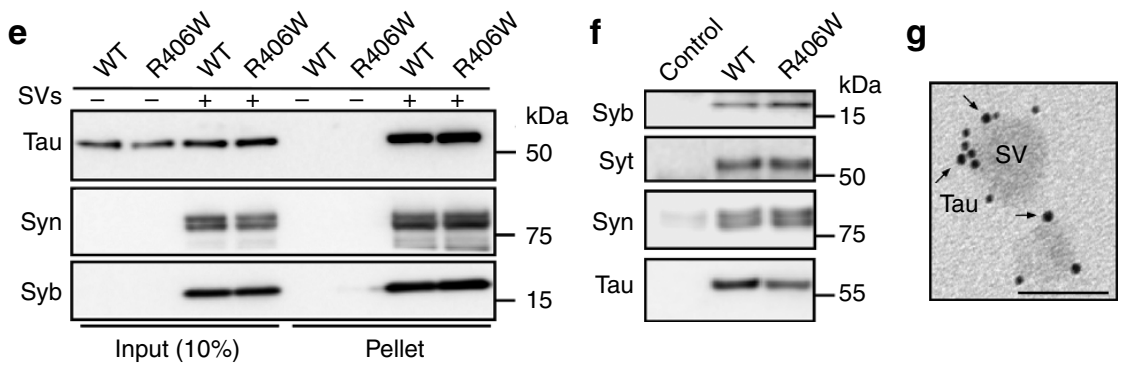

h

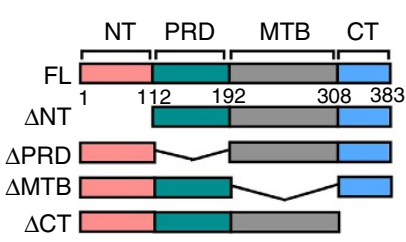

i

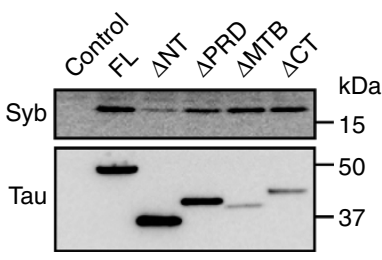

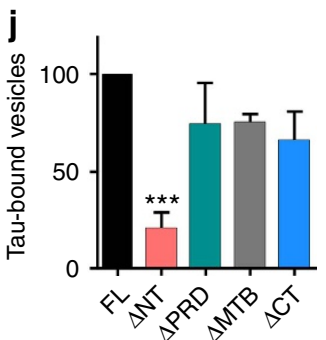

Figure 1 | Tau localizes to presynaptic terminals and binds to synaptic vesicles via its $\mathbf{N}$-terminal domain. (a) Tau and CSP immunolabeling at neuromuscular junctions (NMJs) of Drosophila larvae expressing WT or FTDP-17 pathogenic mutant Tau (R406W, V337M or P301L) under the D42-Gal4 motor neuron driver. Axons (arrowheads) and synaptic boutons (arrows) are indicated. Scale bar, $20 \mu \mathrm{m}$. (b) Quantification of fluorescence intensity of Tau within synaptic boutons (SBs) as ratio to the intensity of axonal Tau. One-way ANOVA, ${ }^{\star \star} P=0.0030,0.0019,0.0041(R 406 \mathrm{~W}, \mathrm{~V} 337 \mathrm{M}, \mathrm{P301L}) \mathrm{n}=10$ (R406W, V337M, P301L) or 12 (WT) NMJs from 5 to 6 animals. Data present mean \pm s.e.m. (c,d) Super-resolution structured illumination microscopy

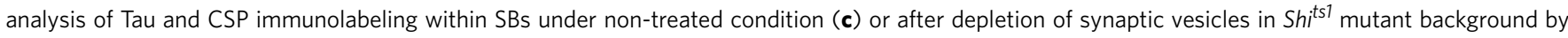
$\mathrm{KCl}$ stimulation at the non-permissive temperature (d). Scale bar, $5 \mu \mathrm{m}$. (e,f) Immunoblots of Tau (anti-His tag) and synaptic vesicle (SV) proteins Synaptobrevin (Syb), Synaptotagmin (Syt) and Synapsin (Syn) from sedimentation assay (e) and co-immunoprecipitation (co-IP) using anti-His antibodies (f) assessing recombinant human Tau binding to purified synaptic vesicles. (g) Electron microscopy imaging of recombinant Tau (probed by Ni-NTANanogold) bound to ultrapure synaptic vesicles in vitro. Scale bar, $50 \mathrm{~nm}$. (h-j) Mapping of the vesicle-binding domain of Tau in vitro by co-IP assay. Truncations of the N-terminal (NT), proline-rich (PRD), microtubule-binding (MTB) or C-terminal (CT) domains of Tau were generated as indicated in the schematic (h). Immunoblots of recombinant Tau domain-truncations and Syb (SV marker) from co-IP using anti-His antibodies (i) and quantification of relative Syb intensity $(\mathbf{j})$. One-way ANOVA, ${ }^{\star \star \star} P=0.002, n=3$ independent experiments. 
Pathogenic Tau restricts synaptic vesicle mobilization. Next we asked if vesicle-associated Tau elicits functional defects at presynaptic terminals. We recorded the spontaneous miniature excitatory junctional potentials (mEJPs) at fly NMJs and found that Tau-expressing animals do not show differences from control animals in mEJP frequency and mean amplitude (Supplementary Fig. $4 \mathrm{a}-\mathrm{d}$ ). We then stimulated motor neurons and recorded excitatory junctional potentials (EJPs). At low-frequency stimulation $(0.2 \mathrm{~Hz})$, the EJP amplitudes measured in controls and mutant Tau-expressing animals are similar (Supplementary Fig. 4e,f). However, during sustained high-frequency stimulation $(10 \mathrm{~Hz})$, pathogenic Tau mutant-expressing animals fail to maintain normal levels of release (Fig. 2a,b). Such a defect could reflect slowed synaptic vesicle reformation by endocytosis $^{40-42}$, a reduced number of synaptic vesicles that participate in release, and/or slower vesicle mobilization ${ }^{43-46}$.

We first assessed synaptic vesicle endocytosis using SynaptopHluorin $(\mathrm{SpH})$, a $\mathrm{pH}$ sensitive GFP fused to the luminal domain of the synaptic vesicle protein Synaptobrevin ${ }^{47}$. The fusion of synaptic vesicles with the presynaptic membrane causes $\mathrm{SpH}$ fluorescence to increase, while vesicle endocytosis and acidification results in a decrease in fluorescence. We did not observe significant differences in the rise and decay of $\mathrm{SpH}$ fluorescence in response to short stimulation paradigms in controls and mutant Tau-expressing animals (Supplementary Fig. 5). In an alternative approach, we also used an exogenously added fluorescent dye (FM 1-43) that is endocytosed into newly formed synaptic vesicles. During mild $3 \mathrm{~Hz}$ stimulation, we did not observe a difference in FM 1-43 uptake in the pathogenic Tau-expressing animals and in controls (Fig. 2c,d). Hence, under these conditions, endocytosis is not affected.

We next assessed synaptic vesicle mobilization during sustained stimulation. We measured $\mathrm{SpH}$ fluorescence during $10 \mathrm{~min}$ of $10 \mathrm{~Hz}$ stimulation in the presence of bafilomycin, a drug that blocks reacidification of newly endocytosed vesicles. Thus, vesicles that fuse at least once during the stimulation paradigm remain fluorescent, revealing the cumulative signals from vesicle fusion over time. Expression of pathogenic Tau mutants slows the vesicle release rate significantly, indicating a smaller active vesicle pool compared to controls or animals expressing wild type Tau (Fig. 2e,f). This effect is not due to a change in total synaptic vesicle pool size, as $\mathrm{NH}_{4} \mathrm{Cl}$ unquenching of all $\mathrm{SpH}$ fluorescence at the synapse does not show significant differences (Fig. 2g). We further confirmed the smaller cycling vesicle pool upon expression of pathogenic Tau by labelling the entire cycling vesicle pool with FM 1-43 dye using prolonged high frequency stimulation $(10 \mathrm{~min}$ at $10 \mathrm{~Hz})$. We observe significantly less dye uptake in pathogenic Tau, but not wild type Tau-expressing NMJs as compared to controls (Fig. 2c,d), indicating fewer labelled vesicles. Note that only mutant Tau, not wild type Tau elicits vesicle cycling defects, again correlating with the increased presynaptic localization of the mutant proteins (Fig. 1).

While our data reveal that the N-terminal domain of Tau binds vesicles, it was also previously described that the proline-rich and microtubule-binding domains of Tau promote actin
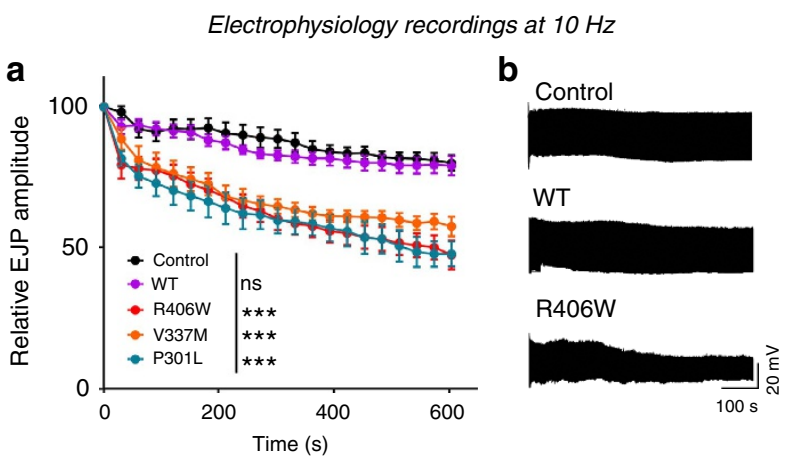

FM1-43 dye loading
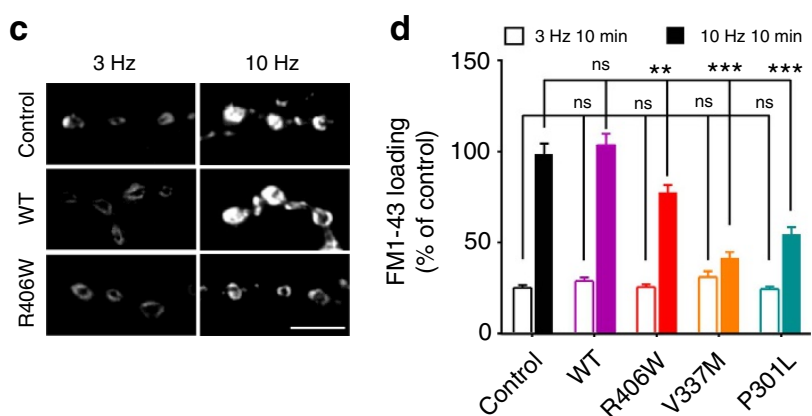

e

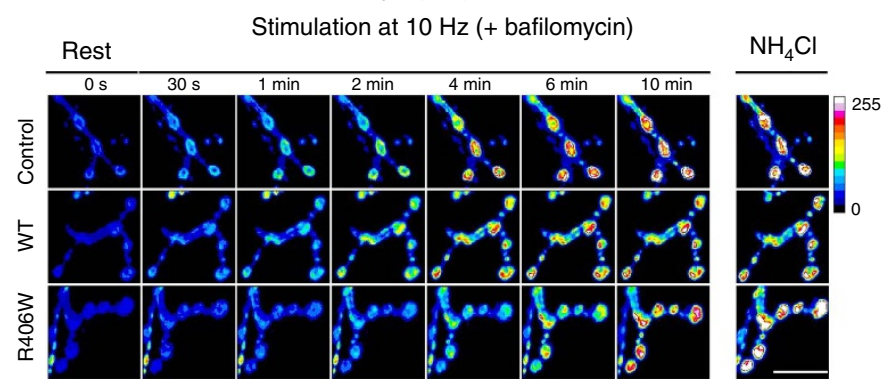

g

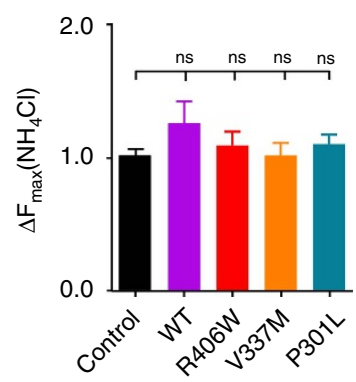

Figure 2 | Pathogenic mutant Tau reduces synaptic transmission and vesicle cycling/release during sustained high-frequency stimulation. Drosophila larvae used in these assays express UAS-Tau (WT, R406W, V337M or P301L) under the D42-Gal4 motor neuron driver. (a,b) Electrophysiological recordings of synaptic transmission during $10 \mathrm{~Hz}$ stimulation for $10 \mathrm{~min}$. Plot of evoked junction potential (EJP) amplitudes (a) and representative traces (b). Two-way ANOVA, ${ }^{\star \star \star} P<0.0001, n=7$ (Control, WT) or 9 (R406W, V337M, P301L) NMJs (animals). (c,d) FM1-43 dye loading with stimulation at $3 \mathrm{~Hz}$ (recycling vesicle pool) or $10 \mathrm{~Hz}$ (reserve vesicle pool) for 10 min. Representative images of FM1-43 dye loading (c) and plot of FM1-43 dye loading intensity (d). One-way ANOVA, ${ }^{\star \star} P=0.0028$ (R406W), ${ }^{\star \star \star} P=0.0001(V 337 M, P 301 L), n=14(\mathrm{WT}, \mathrm{R} 406 \mathrm{~W}, \mathrm{~V} 337 \mathrm{M}, \mathrm{P} 301 \mathrm{~L})$ or 20 (Control) NMJs (animals). Scale bar, $10 \mu \mathrm{m}$. (e-g) Synapto-pHluorin $(\mathrm{SpH})$ responses to stimulation at $10 \mathrm{~Hz}$ with the presence of bafilomycin. Representative images of $\mathrm{SpH}$ responses (e) and plot of fluorescence change $\Delta F$ at ratio to maximal $\Delta F\left(\mathrm{NH}_{4} \mathrm{Cl}\right.$ dequenching) (f). Two-way ANOVA, ${ }^{\star \star \star} P<0.0001, n=7$ (WT, R406W, V337M, P301L) or 11 (Control) NMJs (animals). Plot of maximal $\Delta \mathrm{F}\left(\mathrm{NH}_{4} \mathrm{Cl}\right.$ dequenching) calibrated to control levels (g). One-way ANOVA, ns, not significant. Scale bar, $20 \mu \mathrm{m}$. Data present mean \pm s.e.m. 
polymerization ${ }^{48,49}$. We therefore hypothesized that vesiclebinding and simultaneous F-actin formation by Tau may restrict synaptic vesicle mobility ${ }^{50}$. Accordingly, we find that the presence of Tau increases presynaptic F-actin levels using the genetic probe LifeAct-GFP (Fig. 3a,b). We then performed fluorescence recovery after photobleaching (FRAP) experiments to measure the mobility of synaptic vesicles labelled by Synaptotagmin-GFP within presynaptic boutons. Fluorescence recovery, and thus vesicle mobility, is significantly slower in animals expressing pathogenic mutant Tau compared to controls or animals expressing wild type Tau (Fig. $3 \mathrm{c}-\mathrm{f}$ ). The lower vesicle mobility defect in animals expressing mutant Tau is rescued when we incubate the preparations with the actin depolymerizing drug Latrunculin A to lower presynaptic F-actin levels (Supplementary Figs 6 and 7). These data confirm that increased presynaptic F-actin contributes to the impaired vesicle mobilization. We therefore propose a model in which pathogenic Tau behaves as a vesicle clustering molecule by interacting with vesicles and crosslinking them to F-actin to restrict vesicle mobility and release (Fig. 3g,h)
While pathogenic mutations in Tau lower its microtubule binding affinity, it is well-established that hyperphosphorylation of Tau in $\mathrm{AD}$ and related Tauopathies is the most common cause for impairments in microtubule binding ${ }^{3}$. We thus asked whether hyperphosphorylation of Tau affects synaptic vesicle dynamics in a manner similar to the pathogenic Tau mutants. We used D42-Gal4 to express UAS-Tau ${ }^{\mathrm{E} 14}$ in which 14 putative phosphoSerine/Threonine sites are mutated to glutamate ${ }^{51}$. Similar to the pathogenic mutants of Tau, we find phospho-mimetic Tau ${ }^{\mathrm{E} 14}$ to localize to presynaptic boutons of fly NMJs (Supplementary Fig. 8a,b). The presence of this $\mathrm{Tau}^{\mathrm{E} 14}$ at presynaptic terminals does not affect basal neurotransmitter release characteristics (Supplementary Fig. 8c,d), but it reduced synaptic transmission during a $10 \mathrm{~Hz}$ stimulation train (Supplementary Fig. 8e). Also similar to our observations with expression of pathogenic mutant Tau, FRAP experiments of synaptotagmin-GFP in animals that express $\mathrm{Tau}^{\mathrm{E} 14}$ indicates a lower synaptic vesicle mobility (Supplementary Fig. 8f,h). These data are in line with our model that increased levels of Tau at presynaptic terminals causes synaptic vesicle clustering defects. The defects we observe with

a

Presynaptic F-actin (LifeAct-GFP)

C Synaptic vesicle mobility (FRAP)
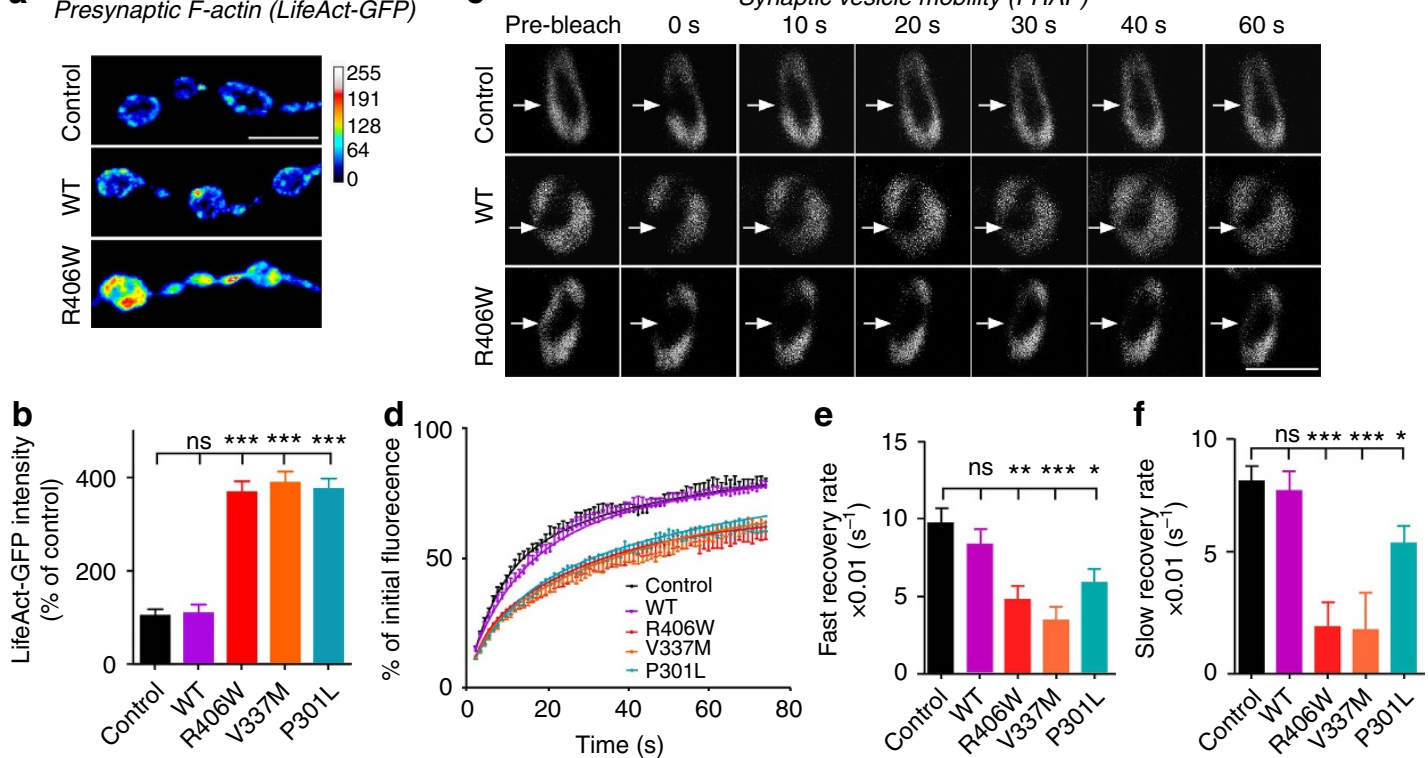

g

The model
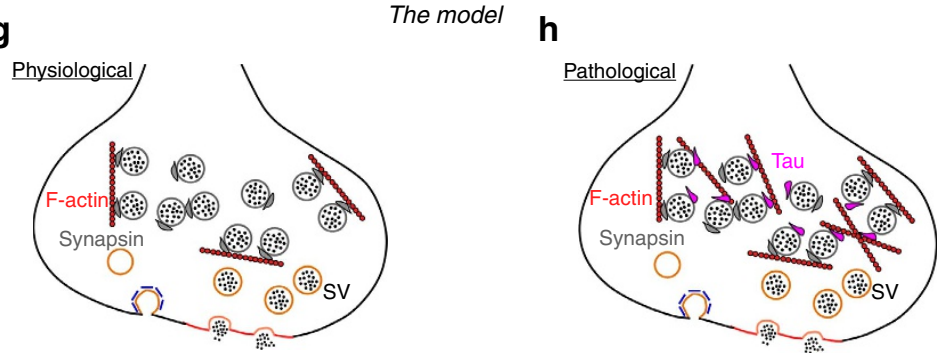

Figure 3 | Pathogenic mutant Tau increases F-actin levels and reduces synaptic vesicle mobility at presynaptic terminals. Drosophila larvae used in these assays express UAS-Tau (WT, R406W, V337M or P301L) under the D42-Gal4 motor neuron driver. (a,b) Immunolabeling of LifeAct-GFP probing for F-actin within synaptic boutons. Representative images of immunolabeling (a) and quantification of LifeAct-GFP intensity (b). One-way ANOVA,

${ }^{\star \star \star} P<0.0001, n=12$ (WT, R406W, V337M, P301L) or 14 (Control) NMJs (two NMJs per animal). Scale bar, $5 \mu \mathrm{m}$. (c-f) FRAP measurement of vesicle mobility within synaptic boutons. Representative images acquired immediately before photobleaching (pre-bleach) and immediately after bleaching at 0-60 s post-bleaching time points (c). Plot of fluorescence recovery (\% of initial fluorescence) over time and fit with double-exponential curve (d). Plot of fast recovery rates calculated from fluorescence recovery curve (e) One-way ANOVA, ${ }^{\star \star} P=0.0011(\mathrm{R} 406 \mathrm{~W}),{ }^{\star \star}{ }^{\star \star} P=0.0001(\mathrm{~V} 337 \mathrm{M}),{ }^{\star} P=0.0158$ (P301L), $n=24,21,22,20,25$ (Control, WT, R406W, V337M, P301L) boutons (3-5 boutons per animal). Plot of slow recovery rates (f). One-way ANOVA, ${ }^{\star} P=0.0001$ (R406W, V337M), ${ }^{\star} P=0.0250$ (P301L), $n=24,21,22,20,25$ (Control, WT, R406W, V337M, P301L) boutons (3-5 boutons per animal). Scale bar, $5 \mu \mathrm{m}$. Data present mean \pm s.e.m. (g,h) Proposed model of Tau clustering synaptic vesicles to F-actin to restrict reserve pool vesicle mobilization and release. 
$\mathrm{Tau}^{\mathrm{E} 14}$ are not because the phosphorylation of Tau affects synaptic vesicle binding. Indeed, the ability of in vitro phosphorylated Tau (by GSK3- $\beta$ and CDK5) to bind synaptic vesicles in co-sedimentation assays is indistinguishable from that of non-phosphorylated Tau (Supplementary Fig. 9). Altogether these data suggest that hyperphosphorylated Tau can bind synaptic vesicles and can impair vesicle mobilization in a manner similar to pathogenic mutant Tau.

Given that the Drosophila models used in the current study ectopically express mutant Tau under a wild type genetic background, we questioned whether competition between mutant Tau and endogenous Tau could contribute to the detected phenotypes of vesicle mobility. To address this question, we analysed $\mathrm{tau}^{-/-}$flies to assess the possible effects of endogenous Tau on vesicle mobilization. Tau knockout flies are viable and fertile, and they do not show defects in basal synaptic transmission at NMJs (Supplementary Fig. 10a-f), nor do they exhibit apparent defects in synaptic transmission during a high frequency $(10 \mathrm{~Hz})$ stimulation paradigm (Supplementary Fig. 10g). Moreover, FRAP experiments at the NMJs did not detect a vesicle mobility phenotype in $\mathrm{tau}^{-/-}$flies (Supplementary Fig. 10h). Altogether, loss-of-function of endogenous Tau does not cause apparent vesicle mobilization phenotypes. Therefore, the impairments of vesicle mobility observed in mutant Tau-expressing animals does not appear to rely on competition with endogenous Tau, and our data support a gain-of-toxic function mechanism of Tau mislocalized to synaptic terminals.

Inhibiting Tau-vesicle binding rescues presynaptic deficits. To test our model that Tau binds and clusters vesicles through its $\mathrm{N}$-terminal domain, we generated transgenic flies that express $\mathrm{N}$-terminally truncated pathogenic mutant Tau $\left(\Delta \mathrm{N} \_\mathrm{R} 406 \mathrm{~W}\right.$, $\Delta \mathrm{N} \_$V337M and $\left.\Delta \mathrm{N} \_\mathrm{P} 301 \mathrm{~L}\right)$ and assessed vesicle mobility and presynaptic function. These $\mathrm{N}$-terminal truncation mutants all localize to presynaptic boutons (Supplementary Fig. 11a,b) and increase presynaptic F-actin levels (Supplementary Fig. 11e) to similar extents as their full-length counterparts. However, $\Delta \mathrm{N}$-pathogenic Tau mutants show a more diffuse localization, distinct from the CSP-labelled 'doughnut-like' synaptic vesicle localization pattern (Supplementary Fig. 11a and c). These data corroborate the inability of $\Delta \mathrm{N}$-Tau to bind vesicles. We confirmed this observation using the $s h i^{t s 1}$ vesicle depletion assay (see above and Fig. 1d). In stimulated $s h i^{t s 1}$ mutants, CSP relocalizes to the plasma membrane but the $\Delta \mathrm{N}$-pathogenic Tau mutants remain diffusely present in the boutons (Supplementary Fig. 11d). Altogether, these data indicate reduced association of $\Delta \mathrm{N}$-Tau with synaptic vesicles in vivo.

To assess vesicle mobility we used the Synaptotagmin-GFP FRAP assay. In contrast to synapses expressing full length pathogenic Tau, synapses expressing $\Delta \mathrm{N}$-pathogenic Tau mutants show Synaptotagmin-GFP fluorescence recovery rates that are similar to those measured in controls (Fig. 4a). Furthermore, animals expressing N-terminally truncated pathogenic Tau mutants also do not show defects in the rate of synaptic vesicle mobilization and in the size of the active synaptic vesicle pool as assessed by $\mathrm{SpH}$ in the presence of bafilomycin (Fig. 4b). Finally, expression of $\mathrm{N}$-terminally truncated Tau mutants does not cause defects in maintaining neurotransmitter release during $10 \mathrm{~Hz}$ stimulation (Fig. 4c). Thus, the N-terminal domain of Tau mediates synaptic vesicle mobilization and the presynaptic defects upon expression of pathogenic Tau in vivo. Furthermore, our data also indicate that increased F-actin levels alone are not sufficient to cluster vesicles and that Tau binding to vesicles is needed as well.

We next assessed Tau-mediated neurodegeneration in the fly brain. Previous work reported vacuolar degeneration in brain sections of Tau transgenic flies ${ }^{38}$. However, our lower Tau expressing lines did not show this defect (Supplementary Fig. 12). Given the synaptic defects we observed in our study we resorted to electron microscopy to reveal presynaptic integrity. Photoreceptors in the fly brain lamina form clean topographic maps, with six presynaptic photoreceptor terminals organizing into one cartridge. In controls and animals expressing wild type Tau, electron micrographs reveal regular shaped cartridges (Fig. 4d,e). In contrast, the organization and morphology of presynaptic terminals are disrupted in animals expressing P301L mutant Tau (Fig. 4f,h). This presynaptic degeneration requires the N-terminal projection of Tau because cartridge morphology and presynaptic integrity is normal in $\Delta$ N_P301L Tau expressing animals (Fig. 4g,h). The data suggest that interfering with vesicle binding prevents mutant Tau-induced presynaptic degeneration in flies.

We further confirmed our findings in rat hippocampal autaptic cultures $^{52}$ (Fig. 5a) by transducing the neurons with AAV expressing either wild type (WT) or mutant Tau (P301L, R406W). As assessed by immunolabeling, both WT and mutant Tau are localized to neurites and similar to our observations in patient samples and Drosophila, mutant Tau (P301L, R406W) is more often present in synapsin-labelled presynaptic puncta (Supplementary Fig. 13). Also consistent with our observations at Drosophila neuromuscular junctions (Supplementary Fig. 4), whole cell voltage clamp recordings of rat neurons expressing wild type Tau or mutant Tau does not show significant differences in basal neurotransmitter release parameters (Supplementary Figs 14 and 15a-d). We then measured neurotransmitter release in response to 10 consecutive highfrequency stimulation trains assessing the ability of vesicle pools to mobilize in order to sustain release $(10 \mathrm{~Hz}$ for $10 \mathrm{~s}$ with $30 \mathrm{~s}$ intervals). Neuronal cultures transduced with AAV-TauWT maintain release efficacy similar to cells transduced with control AAV expressing GFP (Fig. 5b,c). In contrast, neurons expressing pathogenic Tau (P301L or R406W) are unable to maintain release (Fig. 5b,c and Supplementary Fig. 15e,f), in agreement with our observations in Drosophila.

Next, we designed and purified a peptide corresponding to the human Tau N-terminal domain ( $\mathrm{NT}^{\mathrm{Tau}}$, $\mathrm{aa}_{1-112}$ ) fused to a minimal HIV-derived cell-penetrating Tat peptide (Fig. 5e), which is able to compete for synaptic vesicle binding with full-length Tau in vitro (Fig. 5f). Acute treatment of neurons with the Tat-NT ${ }^{\text {Tau }}$ peptide does not affect their basal release characteristics (Supplementary Fig. 14). Similar to our results obtained in Drosophila, we find increased F-actin levels in Tau_P301Lexpressing neurons compared to controls (Supplementary Fig. 16), which is unaffected by treatment with the Tat-NT ${ }^{\text {Tau }}$ peptide. Hence, in agreement with the fly data, interfering with the ability of the N-terminus of Tau to bind synaptic vesicles does not affect Tau-induced actin polymerization at presynaptic terminals.

We next assessed the effect of Tat-NT ${ }^{\text {Tau }}$ treatment on neurotransmitter release during $10 \mathrm{~Hz}$ stimulation trains, and we find that Tat-NT ${ }^{\mathrm{Tau}}$ significantly restores the defect in neurotransmitter release in neurons expressing Tau_P301L, while adding a control Tat-mCherry peptide has no effect (Fig. 5d). In conclusion, both fly and rat neurons display comparable presynaptic defects upon expression of pathogenic Tau, but not expression of wild type Tau, and our data indicate that blocking the ability of the Tau N-terminal domain to interact with synaptic vesicles rescues the presynaptic defects induced by pathogenic Tau.

\section{Discussion}

Our work demonstrates that pathogenic mutant Tau alters presynaptic properties, complementing previous findings that 
a

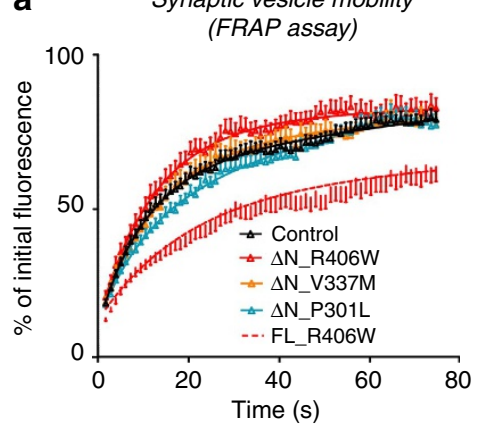

b

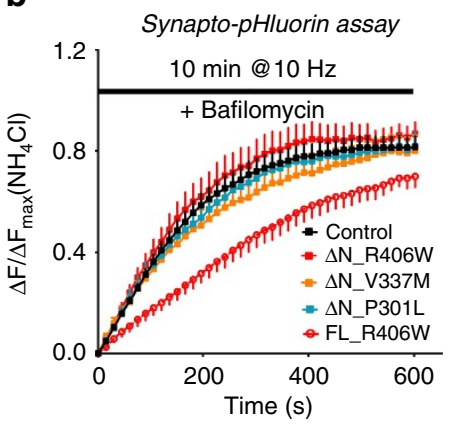

C

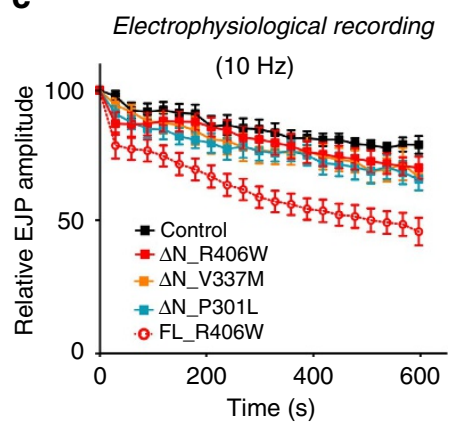

d

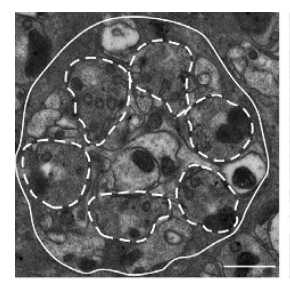

Rh1-Gal4/+
Transmission electron microscopy of the Drosophila lamina

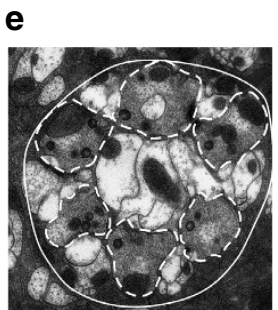

Rh1>Tau_WT f

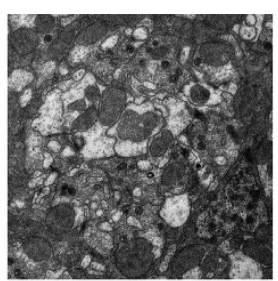

Rh1>Tau_P301L
9

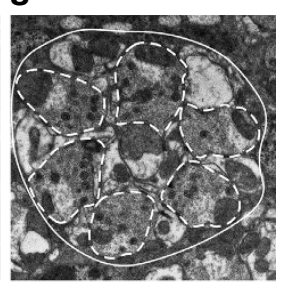

Rh1 $>T^{T a u}{ }^{\Delta N}$ P $301 L$

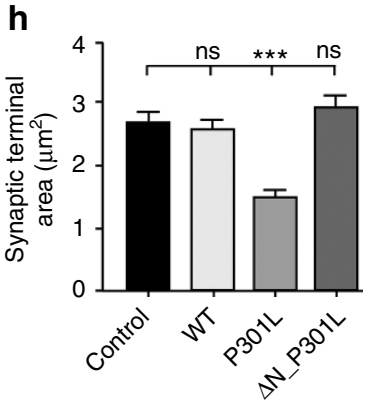

Figure 4 | Interfering with Tau $\mathbf{N}$-terminal-dependent vesicle-binding reverts Tau-induced presynaptic deficits in fly neurons. Drosophila larvae used in (a-c) express UAS-Tau ${ }^{\Delta \mathrm{N}}$ (R406W, V337M or P301L) under the D42-Gal4 motor neuron driver. (a) FRAP measurements of vesicle mobility within synaptic boutons. Fluoresence recovery (\% of initial fluorescence) was plotted over time and fit with double-exponential curves. $n=22$ (R406W), 20 ( $\Delta$ N_R406W, $\Delta$ N_V337M), 24 ( $\Delta$ N_P301L) or 25 (Control) boutons (3-5 boutons per animal). (b) Synapto-pHluorin responses to stimulation at 10 Hz with the presence of bafilomycin. Fluorescence change $\Delta \mathrm{F}$ at ratio to maximal $\Delta \mathrm{F}\left(\mathrm{NH}_{4} \mathrm{Cl}\right.$ dequenching) was plotted over time during the stimulation. Two-way ANOVA, $n=7$ (R406W, $\Delta$ N_R406W, $\Delta$ N_V337M, $\Delta$ N_P301L),9 (Control) NMJs (animals). (c) Electrophysiological recordings of EJP amplitudes during stimulation at $10 \mathrm{~Hz}$. Two-way ANOVA, $n=7$ (R406W, $\Delta \mathrm{N} \_R 406 \mathrm{~W}$ ), 8 ( $\Delta \mathrm{N} \_V 337 \mathrm{M}, \Delta \mathrm{N} \_\mathrm{P} 301 \mathrm{~L}$ ), or 9 (Control) NMJs (animals). (d-h)

Transmission electron microscopy (TEM) of lamina sections of 9-day-old flies expressing UAS-Tau (WT, P301L or $\Delta$ N_P301L) under the late-onset retinal driver Rhodopsin1-Gal4 (Rh1). In control Rh1-Gal4/ + animals (d), six photoreceptor synaptic terminals (outlined in dashed white lines) are converged in a 'cartridge' (outlined in white line). Disrupted synaptic terminal organization and morphology are detected in Rh1> Tau_P301L (f) flies, whereas no obvious abnormalities at the synaptic terminals of Rh1>Tau_WT (e) and Rh1 > Tau ${ }^{\Delta N} \_$P301L (g) flies. Scale bar, $1 \mu$ m. (h) Quantification of the synaptic terminal area based on the electromicrographs. One-way ANOVA, ${ }^{\star \star \star} P=0.0001$. $n=5$ animals per genotype. Data present mean \pm s.e.m.

expression of mutant Tau affects postsynaptic functions ${ }^{18,21}$. Both wild type and pathogenic mutant Tau can bind synaptic vesicles to a similar extent in vitro, but expression of the mutant form leads to increased localization of Tau to presynaptic terminals. In addition to pathogenic mutations, abnormal phosphorylation of Tau in $\mathrm{AD}$ also results in the dissociation of Tau from microtubules. At fly NMJs, phospho-mimetic Tau ${ }^{\text {E14 }}$ behaves similar to pathogenic mutant Tau, as it mislocalizes to presynaptic terminals and impairs vesicle mobilization. Our data also show that pathologically phosphorylated Tau binds to synaptic vesicles in vitro. This again supports the notion that phosphorylation or clinical mutations drive Tau to the synapse where it comes in contact with vesicles, but does not directly affect the binding affinity of Tau to vesicles; this also makes sense in light of the fact that Tau phosphorylation sites (mostly within the proline-rich and C-terminal domains) and sites of diseasecausing mutations in Tau (clustering around the microtubule binding domain) do not overlap with the vesicle binding domain (the N-terminal projection) that we identified here. Therefore, we propose that this mechanism may be common to various Tauopathies associated with abnormal phosphorylation or mutations of Tau.

We propose a model in which when Tau is present presynaptically, it binds to vesicles with its $\mathrm{N}$-terminal domain and polymerizes presynaptic actin using its proline-rich and microtubule-binding domains. Tau thereby crosslinks synaptic vesicles by binding vesicles and actin, and slows their mobilization, lowering synaptic transmission during intense stimulation. We suggest a multi-step mode of action in which disease conditions lead to the dissociation of Tau from microtubules, followed by an early soluble phase where the protein induces pathological synaptic dysfunction presynaptically as we show here. Indeed, recent studies in Tau transgenic Drosophila and mice both detected early synaptic dysfunction preceding pathological aggregation and neurodegeneration ${ }^{23,53}$, and we propose here that the pathway identified in the current work may underlie early presynaptic dysfunction, triggering a cascade of events ultimately leading to synaptic and neuronal loss. We now show that Tau $\mathrm{N}$-terminal-dependent vesicle binding is a key mechanism that induces presynaptic defects in the absence of tangle formation. Our work also suggests that interfering with Tau-N-terminal dependent vesicle-binding could be exploited therapeutically to prevent these aspects of presynaptic pathology.

The discovery of a function for the $\mathrm{N}$-terminal projection dissociates the vesicle binding and actin polymerization role of pathogenic Tau at synapses. The N-terminal domain of Tau is dispensable for actin polymerization because $\Delta \mathrm{N}$-pathogenic Tau mutants localize to presynaptic terminals and still support the formation of excess F-actin at synapses. These results also indicate that Tau-induced actin polymerization at synapses alone is not sufficient to measurably hinder vesicle mobilization; rather, the direct binding of Tau to synaptic vesicles is also needed. Notably, Tau also dimerizes and multimerizes ahead of tangle formation and while not studied further here, this activity could 
a

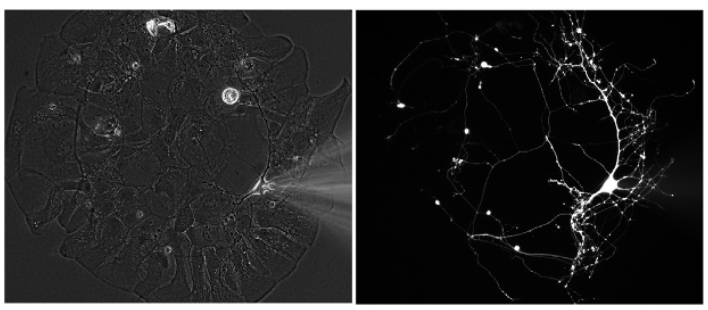

C
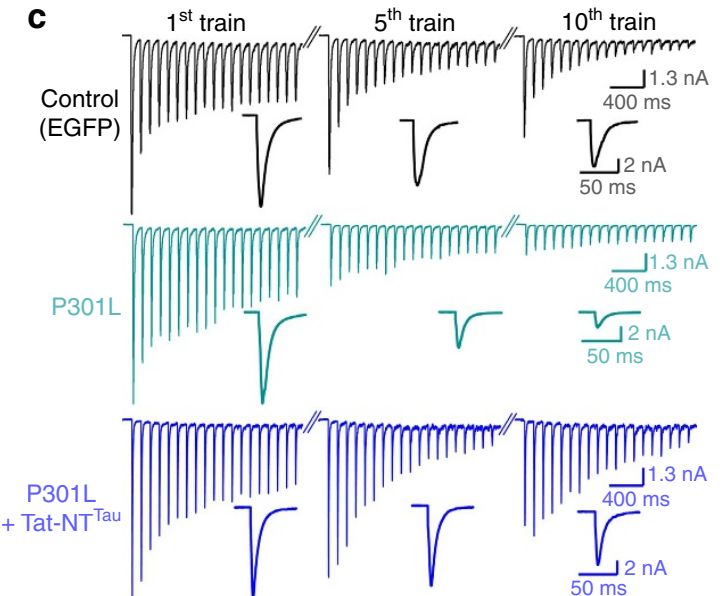

b
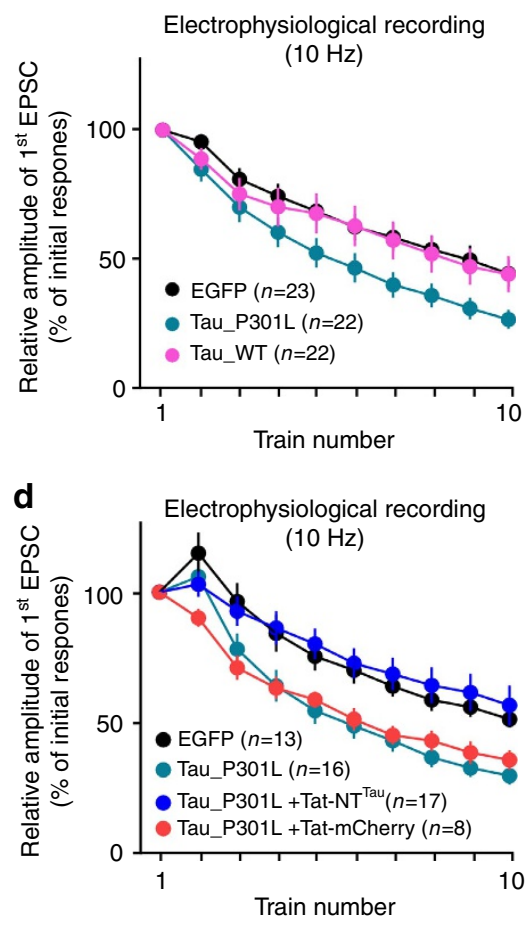

e

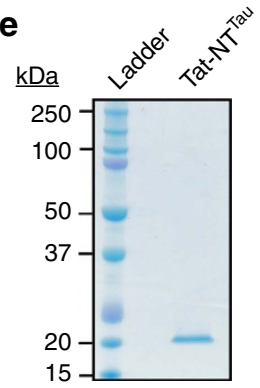

$\mathbf{f}$

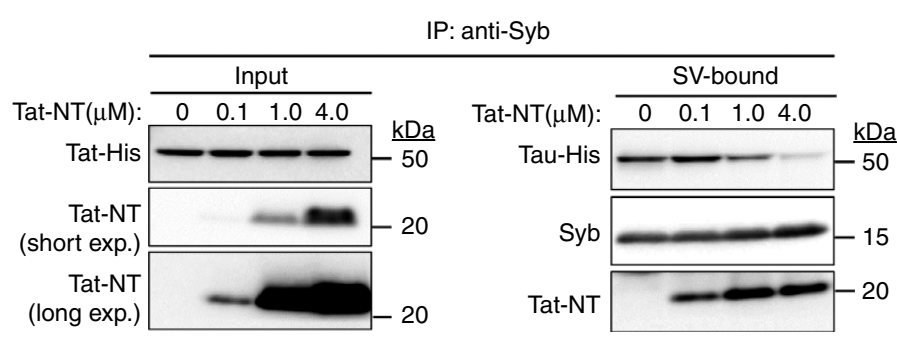

Figure 5 | Interfering with Tau $\mathbf{N}$-terminal-dependent vesicle-binding rescues Tau-induced presynaptic deficits in cultured rat hippocampal neurons. Autaptic rat hippocampal neuronal cultures were transduced with AAV viral vectors expressing GFP or Tau variants. (a) Autaptic neuronal culture transduced with AAV-EGFP is visualized by bright-field illumination (left) and GFP fluorescence (right). (b-d) Electrophysiological recordings using patch clamp in response to 10 consecutive high frequency stimulation trains $(10 \mathrm{~Hz}$ for $10 \mathrm{~s}$ with $30 \mathrm{~s}$ interval). The representative traces are shown in (c) and the relative first EPSCs were plotted to train numbers $(\mathbf{b}, \mathbf{d})$. In (d), Tau ${ }^{\mathrm{P} 301 \mathrm{~L}}$ expressing neurons were acutely treated with $5 \mu \mathrm{M}$ Tat-NT Tau or control Tat-mCherry peptides before patch clamp recordings. The experiments were independently repeated at least three times and the number of neurons recorded is indicated in the graph. Data present mean \pm s.e.m. (e) Colloidal coomassie staining of purified Tat-NTTau peptide; NT Tau corresponds to the $\mathrm{N}$-terminal domain (aa 1-113) of Tau. (f) Tat-NTTau peptide competes with full-length Tau for vesicle binding in vitro. Intact synaptic vesicles (SVs) were immobilized to Dynabeads using anti-Synaptobrevin 2 (Syb) antibodies. Following immobilization, SVs were incubated with recombinant full-length Tau $(40 \mathrm{nM})$ with or without the presence of $0.1,1.0$ or $4.0 \mu \mathrm{M}$ purified Tat-NTTau peptide. Immunoblots were probed for full-length Tau, Tat-NTTau and the synaptic vesicle marker Syb. Note that Tat-NT Tau reduced the amount of full-length Tau bound to SVs in a dose-dependent manner.

potentially also add to the ability of Tau to impede with vesicle mobility. Interestingly, a similar pathogenic function, the direct cross-linking of vesicles by multimerization, has been suggested for $\alpha$-synuclein ${ }^{54,55}$, a protein that aggregates in Lewy body disease and Parkinson's disease, suggesting that Tau and $\alpha$-synuclein may harbour overlapping effects on presynaptic function.

Our findings suggest that Tau employs a similar mode of vesicle immobilization as Synapsins, which also bind vesicles and $\mathrm{F}$-actin to keep vesicles in a reserve pool ${ }^{50}$. Consistent with this function, we observed a defect to maintain normal levels of neurotransmitter release in fly and rat neurons expressing pathogenic Tau. This neurotransmitter release defect is similar to that seen upon overexpression of Synapsin, as in different model synapses, expression of Synapsin has been shown to result in a decline of neurotransmission in response to strong stimulation trains ${ }^{56,57}$. There are previous studies showing reduced amplitudes of mEPSCs in primary neurons expressing mutant Tau at DIV 22-30 (refs 21,58). In our study, at much earlier time points (DIV 11-13), we did not detect such a change in mEPSCs. The discrepancy is likely due to the postsynaptic accumulation of mutant Tau in older neuronal cultures, in which dendritic spines are more mature and relatively stable 59,60 Conversely, the dendritic protrusions in younger neurons are rather transient and highly dynamic ${ }^{59,60}$. Thus we surmise our electrophysiological recordings at DIV 11-13 mainly reflect the effects of Tau on presynaptic vesicle release, where we did not detect a deficit at basal level. There are also electrophysiological 
studies performed in Tauopathy mouse models, but mostly at a stage where substantial synapse and neuron loss had already occurred and these studies also often focused on the post-synaptic role of $\mathrm{Tau}^{18,61-63}$. Consistent with our observations that expression of Tau lowers neurotransmitter release efficiency, more recent studies in mice overexpressing Tau_P301L indicated early stage defects and show a loss of synaptic input before synapse loss in intact neocortical pyramidal cells as well as alterations in the probability of neurotransmitter release in entorhinal cortex ${ }^{19,23}$. While a connection between vesicle mobilization defects and neuronal death awaits further investigation, it is conceivable that a defect to maintain normal release contributes to the early synaptic defects seen in neurodegenerative disease. The strength of the defects we observed are also subtle enough to explain the occurrence of disease symptoms only later in life. In addition, in humans, long lasting high-frequency activity has been observed during cognitive tasks ${ }^{64-66}$, suggesting that Tau-induced vesicle mobilization defects and the inability to maintain normal levels of neurotransmitter release could potentially contribute to cognitive decline in dementia.

A number of recent studies show that Tau N-terminal fragments, including a $17-\mathrm{kDa}$ (Tau44-230) fragment and a $35-\mathrm{kDa}$ (Tau1-391) fragment, are elevated in the brains of Tauopathy patients ${ }^{58,67}$. These two fragments are generated by Calpain and Caspase- 2 cleavage of Tau, and they are shown to be neurotoxic in cellular and animal models ${ }^{58,67}$. The $35-\mathrm{kDa}$ fragment acts similar to soluble pathogenic Tau, as it binds less efficiently to microtubules and missorts to dendritic spines to impair synaptic function ${ }^{58}$. It remains to be assessed, but it is likely, that the $35-\mathrm{kDa}$ fragment also missorts to presynaptic terminals, where it can bind to synaptic vesicles and simultaneously promote actin polymerization, as it contains the required domains for both vesicle- and actin-binding. Whether the $17-\mathrm{kDa}$ fragment causes synaptic toxicity remains unknown, but it has been shown to inhibit aggregation of full-length $\mathrm{Tau}^{67}$. Thus the presence of the $17-\mathrm{kDa}$ fragment may potentially lead to an increase of soluble Tau under disease conditions, and subsequently more Tau missorting to the synapse. Our work shows that increased soluble Tau at presynaptic terminals impairs vesicle dynamics, which could be a possible mechanism that contributes to the toxicity of these $\mathrm{N}$-terminal fragments. Future work should assess these possibilities.

Our work indicates that targeting the $\mathrm{N}$-terminal domain of Tau is sufficient to alleviate the presynaptic defects induced by the expression of pathogenic Tau. Indeed, expression of mutant Tau proteins lacking the $\mathrm{N}$-terminal projection does not elicit presynaptic defects anymore. We therefore reasoned that tools that compete with Tau for vesicle binding would be beneficial. Treating rat neurons that express Tau_P301L with a membrane permeable N-terminal Tau domain (Tat-NT ${ }^{\text {Tau }}$ peptide), shown to compete for synaptic vesicle binding, rescues the pathological Tau-induced inability to maintain neurotransmitter release. Our work provides proof-of principle that it is possible to target the ability of Tau to interact with vesicles to rescue presynaptic defects elicited by the excessive presynaptic localization of pathogenic Tau. These findings also pave the way for the development of more specific tools with higher affinity and specificity. In relation to this, while short-term exposure to the current $\mathrm{N}$-terminal peptide itself did not elicit obvious neuronal toxicity, it will also be essential to assess if longer exposure of tools that inhibit Tau vesicle binding affect aspects of presynaptic or other neuronal function for their therapeutic potential. Nonetheless, our work reveals a new aspect of Tau biology and suggests there is a window to tackle the presynaptic defects induced by pathological Tau.

\section{Methods}

Drosophila genetics. All Drosophila melanogaster stocks and experimental crosses were kept on standard corn meal and molasses food. Stocks were kept at room temperature. Experimental crosses involving UAS/GAL4 bipartite expression were kept at $25^{\circ} \mathrm{C}$. All UAS-human Tau (0N4R) flies used in this work were generated by insertion of transgene into a same genomic locus (68A4) and were expressed under the motor neuron-specific D42-Gal4 driver. The UAS-Tau_WT and UAS-Tau_R406W fly lines were obtained as gifts from lab of Dr. Guy Tear. The UAS-Tau_V337M and UAS-Tau_P301L fly lines were generated in this current work. Additional stocks used in this study include UAS-Synapto-pHluorin, UAS-LifeAct-GFP (BSC 57326) and UAS-synaptotagmin-eGFP (BSC 6925) from Bloomington Stock Center. UAS-hTau_WT(H), UAS-hTau_R406W (H) and UAS-hTau_E14 (III) fly lines were obtained as gifts from lab of Dr. Mel Feany. Tau knockout fly line was generated by genome editing ${ }^{68}$.

Synapto-pHluorin imaging at fly NMJs. Third-instar Drosophila larvae were dissected in $\mathrm{Ca}^{2+}$-free HL3 saline $(110 \mathrm{mM} \mathrm{NaCl}, 5 \mathrm{mM} \mathrm{KCl}, 10 \mathrm{mM} \mathrm{NaHCO}$, $5 \mathrm{mM}$ HEPES, $30 \mathrm{mM}$ sucrose, $5 \mathrm{mM}$ trehalose, $10 \mathrm{mM} \mathrm{MgCl}_{2}, \mathrm{pH}$ 7.2) and then incubated in HL3 supplemented with $1 \mathrm{mM}$ calcium and $500 \mu \mathrm{M}$ 1-Naphthylacetyl spermine trihydrochloride (Sigma) to prevent muscle contractions ${ }^{69}$. Motor nerves were stimulated at $\times 2$ threshold using an Axoclamp 900A amplifier, and images were captured though a water-dipping objective $(\times 40,0.8$ N.A. $)$ using a cooled CCD camera (Andor Clara DR-328G-CO1-SIL) mounted on a Nikon ECLIPSE FN1 microscope. Images were analysed using NIS-Elements AR3.2 software. To assess vesicle exocytosis and endocytosis, motor nerves were stimulated at $10 \mathrm{~Hz}$ for $5 \mathrm{~s}$ and images were captured at $900 \mathrm{~ms}$ intervals before, during and after stimulation. The fluorescence changes $(\Delta \mathrm{F} s \mathrm{sH})$ reflect the intensities of boutonic fluorescence at corresponding time points subtracting the basal fluorescence intensities before stimulation. To assess endocytosis, $\Delta \mathrm{F}$ was normalized by ratio to peak $\Delta \mathrm{F}$ and the fluorescence decays were fitted in two-phase curves for fast and slow time constants to assess the rates of endocytosis. To assess vesicle release during sustained stimulation, $0.5 \mu \mathrm{M}$ bafilomycin (Merck Millipore) were added into the incubation bath of dissected larva. Motor nerves were stimulated at $10 \mathrm{~Hz}$ for $10 \mathrm{~min}$ and images were captured at $15 \mathrm{~s}$ intervals. After stimulation, $50 \mathrm{mM}$ $\mathrm{NH}_{4} \mathrm{Cl}$ (pH7.4) was added to dequench $\mathrm{spH}$ fluorescence signals. The ratios of $\Delta \mathrm{F} / \Delta \mathrm{Fmax}\left(\mathrm{NH}_{4} \mathrm{Cl}\right)$ were plotted to corresponding time points. In all experiments the NMJs at muscles 12 and 13 in segments A2-A4 were selected for imaging. Data are expressed as average \pm s.e.m.

FM1-43 dye labelling. Third-instar larvae were dissected in $\mathrm{Ca}^{2+}$-free HL3 saline on Sylgard-coated plates. Motor nerves were stimulated at $\times 2$ threshold using an Axoclamp 900A amplifier to load FM1-43 dye ( $4 \mu \mathrm{M}$; Molecular Probes) in HL3 solution supplemented with $1 \mathrm{mM} \mathrm{Ca}^{2+}$. FM dye loading of NMJs at muscle 12/13 in segments A2-A4 was imaged using a $\times 601.0$ N.A. water immersion lens on a Nikon fluorescent microscope. The microscope filter was set for FM1-43 emission and excitation.

Electrophysiological recording at fly NMJs. Intracellular voltage recordings from third-instar larval muscle 12 in segment A2 or A3 were performed using $\sim 20 \mathrm{M} \Omega$ sharp electrodes and stimulation at $\times 2$ threshold. EJPs and spontaneous miniature junctional potentials (mEJPs) were recorded with an Axoclamp 900A amplifier digitized using a Digi-data $1440 \mathrm{~A}$ and stored using pClamp 10.2 software (Molecular Devices). For quantification of $10 \mathrm{~Hz}$ recordings, EJP amplitudes were binned per $30 \mathrm{~s}$ and averages are normalized to the amplitude measured in the first 15 stimuli.

Fluorescence recovery after photo-bleaching assay. Experiments were performed on third-instar larval fillets dissected in HL3 saline on sylgard-coated plates. For FRAP analysis, all images were captured within one hour after dissection of the larval, using a Nikon A1R confocal laser microscope equipped with a $\times 601.0$ N.A. water immersion objective. The vesicle dynamics were recorded from type Ib boutons on NMJs at muscles 12 and 13, with 3-5 boutons from the same larvae used. Images were acquired at $1.12 \mu$ s per pixel with a pinhole of one airy unit and a resolution of $512 \times 512$. A region of interest (ROI) $24 \times 30$ pixels was selected on the digital image for photo-bleaching. Four baseline scans were performedusing $5 \%$ of full laser power. Immediately before the fifth scan, the laser power were increased to $95 \%$ of maximal and rapidly iterated the ROI for nine times. After the photo-bleaching, the laser was returned to $5 \%$ of maximal power to complete the remaining scans. Fluorescence intensity of the bleached region, the selected background region and the reference bouton were analysed using NIS-Elements AR3.2 software with the Time Series Analyzer plug-in. The recovery curves were fitted a double exponential curve, which was further used to to account for the two-phasefluorescence recovery of the initial faster recovery immediately after bleaching and the later plateau observed with respect to vesicle mobility. For FRAP experiments performed with Latrunculin A (LatA) treatment, Drosophila larvae were dissected in HL3 buffer then bathed in HL3 buffer with $10 \mu \mathrm{M}$ LatA (sea sponge origin, Sigma L5163) for 5-7 min, followed by FRAP measurements in the same buffer containing Lat A. Live imaging was carried out in $<30 \mathrm{~min}$ following onset of Lat A treatment. 
Vesicle depletion in shits1 mutant background. Third-instar larvae were dissected in $\mathrm{Ca}^{2+}$-free HL3 buffer on a sylgard dish at room temperature. The sylgard dish with the dissected preparation in $\mathrm{Ca}^{2+}$-free $\mathrm{HL} 3$ buffer was placed in a $34^{\circ} \mathrm{C}$ oven, and the buffer was replaced by pre-warmed $\mathrm{Ca}^{2+}$-free HL3 buffer $\left(34^{\circ} \mathrm{C}\right)$. After allowing 3-5 min for temperature to equilibrate, the preparation was stimulated in pre-warmed high $\mathrm{K}^{+}$(HL3 buffer supplemented with $60 \mathrm{mM} \mathrm{KCl}$ and $1.5 \mathrm{mM} \mathrm{Ca}^{2+}$ ) at $34^{\circ} \mathrm{C}$ for $10 \mathrm{~min}$. This stimulation was followed by $5 \mathrm{~min}$ incubation in pre-warmed $\mathrm{Ca}^{2+}$-free $\mathrm{HL} 3$ at $34^{\circ} \mathrm{C}$. The samples were then fixed in pre-warmed $4 \%$ PFA at $34^{\circ} \mathrm{C}$ for $10-15$ min and proceed with washing and immunofluorescence staining. Images were obtained with a Zeiss ELYRA super resolution microscope.

Immunohistochemistry. Third-instar Drosophila larvae were dissected in fresh HL-3 solution and fixed in $3.7 \%$ formaldehyde for $20 \mathrm{~min}$ at room temperature. After washing with PBS, tissue was permeabilized with PBS containing $0.4 \%$ TritonX-100 (PBT) for $1 \mathrm{~h}$, followed by blocking in $1 \%$ BSA for $1 \mathrm{~h}$ at room temperature. Immunostaining was performed with the following primary antibodies: DAKO anti-total tau rabbit pAb (DAKO, A0024, 1:1,000), anti-CSP2 mouse $\mathrm{mAb}$ (DSHB, 6D6, 1:1,000) and anti-GFP rabbit pAb (Invitrogen, A-11122, 1:1,000) for probing LifeAct-GFP. The secondary antibodies used were: goat antirabbit Alexa 488 (Invitrogen, 1:5,000) and goat anti-mouse Alexa 555 (Invitrogen, 1:5,000). Following antibody labelling, the preparations were washed in PBT and mounted in Vectashield (Vector Laboratories). Samples were visualized on a Nikon confocal microscope or a Zeiss ELYRA super resolution microscope.

Immunoblotting. All protein samples were reduced in $\times 1$ lithium dodecyl sulfate (LDS) sample buffer (Invitrogen) supplemented with $1 \% \beta$-mercaptoethanol for $10 \mathrm{~min}$ at $70^{\circ} \mathrm{C}$. Proteins were separated on NuPAGE Novex $4-12 \%$ Bis-Tris polyacrylamide gels (Invitrogen) in MOPS buffer and then transferred to nitrocellulose membrane using the Trans-Blot Turbo transfer system (BioRad). For immunoblotting, membranes were blocked in Tris-buffered saline $+0.05 \%$ Tween20 (TBST) with $5 \%$ milk powder for 1 hour at room temperature before incubation with primary antibodies diluted in blocking buffer. The following primary antibodies were used in this study: DAKO against total Tau (DAKO, A0024, 1:1,000), anti-Synaptobrevin 2 (Synaptic Systems Clone 69.1, 1:1,000), anti-Synaptotagmin (DSHB, Asv48, 1:1,000), anti-Synapsin (Merck Millipore AB1543P, 1:1,000), antiSynaptophysin (Synaptic Systems Clone 7.2, 1:1,000) and anti-Tubulin (DSHB, E7, 1:5000). After incubation with primary antibodies, HRP-conjugated species-specific secondary antibodies were added at a concentration of 1:10,000 for 1 hour at room temperature. Signal was detected using the Western Lightning Plus ECL kit (Perkin Elmer) and imaged on a Fuji-Film digital imaging system. Uncropped immunoblot images for all blots in this study can be found in Supplementary Fig. 17.

Ultrapure synaptic vesicle purification from rat brains. Synaptic vesicles were purified using differential centrifugation and size exclusion chromatography ${ }^{70}$. The whole procedure was carried out on ice or at $4{ }^{\circ} \mathrm{C}$ to minimize proteolysis. Briefly, 20 rat brains weighing between 150 and $180 \mathrm{~g}$ werecollected, and thetissue was homogenized in a motor driven glass-teflon homogenizer at 900 r.p.m., using $240 \mathrm{ml}$ ice-cold sucrose buffer ( $320 \mathrm{mM}$ sucrose, $4 \mathrm{mM}$ HEPES ( $\mathrm{pH}$ 7.4, $\mathrm{NaOH}$ )) supplemented with $0.2 \mathrm{mM}$ phenylmethylsulfonylfluoride (PMSF) and $1 \mathrm{mg} \mathrm{ml}^{-1}$ pepstatin $\mathrm{A}$. The homogenate $(\mathrm{H})$ was centrifuged for $10 \mathrm{~min}$ at $800 \mathrm{~g}$ Av to yield a pellet (P1). The supernatant was further centrifuged for $15 \mathrm{~min}$ at $12,000 \mathrm{~g} \mathrm{Av}$ to generate the $\mathrm{P} 2$ pellet, which was washed twice with sucrose buffer, yielding a clean synaptosome pellet $\left(\mathrm{P}^{\prime}\right)$. The synaptosomal pellet was resuspended in $24 \mathrm{ml}$ sucrose buffer and lysed by addition of 9 volumes distilled $\mathrm{H}_{2} \mathrm{O}$, followed by homogenization. Five mM HEPES ( $\mathrm{pH} 7.4, \mathrm{NaOH}$ ) was added, supplemented with $0.2 \mathrm{mM}$ PMSF and $1 \mathrm{mg} \mathrm{ml}^{-1}$ pepstatin A. This lysate was then centrifuged for $20 \mathrm{~min}$ at $32,500 \mathrm{~g} \mathrm{Av}$ giving a pellet (LP1) and supernatant (LS1). The supernatant was further centrifuged for $2 \mathrm{~h}$ at 230,000 gAv, yielding a crude synaptic vesicle pellet (LP2). This crude synaptic vesicle pellet was resuspended in $40 \mathrm{mM}$ sucrose and further centrifuged for $4 \mathrm{~h}$ at $82,500 \mathrm{gAv}$ on a continuous sucrose density gradient (50-800 mM sucrose). Vesicles were collected from the gradient and subjected to size-exclusion chromatography on controlled pore glass beads $(300 \mathrm{~nm}$ diameter), equilibrated in $300 \mathrm{mM}$ glycine, $5 \mathrm{mM}$ HEPES (pH 7.4, KOH), to separate synaptic vesicles from residual myelin and larger membrane contaminants. Synaptic vesicles were pelleted by centrifugation for $2 \mathrm{~h}$ at 230,000 $\mathrm{g} \mathrm{Av}$ and resuspended in HB150 buffer ( $150 \mathrm{mM} \mathrm{KCl}, 1 \mathrm{mM}$ DTT, $25 \mathrm{mM}$ HEPES ( $\mathrm{pH} 7.4, \mathrm{KOH})$ ) by homogenization. Aliquotes of the resuspended synaptic vesicles were snap frozen in liquid nitrogen. Electron microscopy (EM) and western blotting were applied to assess the purity of synaptic vesicles. For EM analysis, synaptic vesicles were absorbed to formvar-coated grids, fixed with $1 \%$ paraformaldehyde, quenched with $20 \mathrm{mM}$ glycine and immunostained for synaptophysin (Clone G95). Protein A-gold was used for detection. After counterstaining with $1 \%$ uranylacetate, samples were imaged using a CM120 electron microscope, equipped with a TemCam 224A CCD camera. 95\% plus of structures were immunopositive for synaptophysin and were $40-50 \mathrm{~nm}$ in diameter, which is the typical size for synaptic vesicles. For western blotting, the protein concentration of collected fractions was determined using a modified Lowry assay using BSA as a standard. Approximately $10 \mu \mathrm{g}$ total protein was separated using a tricine based gel system, and then transferred to nitrocellulose membrane using standard semi-dry techniques. Membranes were blocked and incubated with primary antibodies (anti-synaptophysin, clone 7.2, Synaptic Systems; anti-NMDA-Receptor 1, clone M68, Synaptic Systems) overnight at $4{ }^{\circ} \mathrm{C}$. HRP-conjugated secondary antibodies were then added for one hour at room temperature. Blots were developed using Western Lightning chemiluminescence reagents and images acquired using a Fuji-LAS reader. As expected, the purified synaptic vesicle fraction showed enrichment of synaptophysin and depletion of NMDA-R1.

Synaptic vesicle sedimentation assay. To perform the co-sedimentation assay, $500 \mathrm{ng}$ of freshly purified recombinant human Tau-His was incubated with an excess amount of ultrapure synaptic vesicles (equivalent to $20 \mu \mathrm{g}$ of protein) prepared from rat brains in synaptic vesicle binding buffer (4 mM HEPES $\mathrm{pH}$ 7.4, $5 \mathrm{mM}$ Tris- $\mathrm{HCl} \mathrm{pH} \mathrm{7.4,220} \mathrm{mM} \mathrm{glycine,} 0.1 \% \mathrm{BSA}$, protease inhibitors) at $4{ }^{\circ} \mathrm{C}$ for $2 \mathrm{~h}$ in a volume of $100 \mu \mathrm{l}$. After $2 \mathrm{~h}$ of incubation, vesicles were pelleted by ultracentrifugation at $400,000 \mathrm{~g}$ for $30 \mathrm{~min}$. Pellets were then re-suspended and reduced in LDS sample buffer. The presence of recombinant human Tau-His in the synaptic vesicle pellet was assessed by immunoblotting using anti-His antibodies.

Transmission electron microscopy. Adult fly heads were dissected and immediately fixed in $4 \%$ paraformaldehyde and $2 \%$ glutaraldehyde in $0.1 \mathrm{M} \mathrm{Na}$-Cacodylate buffer ( $\mathrm{pH}$ 7.4) for $2 \mathrm{~h}$ at room temperature. Samples were further fixed at $4^{\circ} \mathrm{C}$ overnight, and then washed with $0.1 \mathrm{M} \mathrm{Na}$-Cacodylate, $\mathrm{pH}$ 7.4, and subsequently osmicated with $2 \%$ osmium $\left(\mathrm{OsO}_{4} / \mathrm{Na}\right.$-Cacodylate buffer). After dehydration in an ascending series of ethanol solutions and staining in $4 \%$ aqueous uranyl acetate solution the specimen were embedded in Agar 100 (Laborimpex; Agar Scientific). Ultrathin sections $(70 \mathrm{~nm})$ of the retina and the lamina were collected on grids (Laborimpex; Agar Scientific) coated with Butvar and imaged on a JEM 1400 transmission electron microscope (JEOL) at $80 \mathrm{kV}$ with a bottom mounted camera (Quemasa; 11 megapixels; Olympus) running iTEM 5.2 software (Olympus).

Rat neuronal cultures. Wistar rat embryos (Janvier Labs) of embryonic day 18 (E18) were dissected in hanks buffered salts solution (HBSS; Sigma) buffered with $7 \mathrm{mM}$ HEPES, and the hippocampi were collected in the dissection buffer. After removal of the meninges, hippocampi were minced and incubated in $0.25 \%$ trypsine in HBSS for $15-20 \mathrm{~min}$ at $37^{\circ} \mathrm{C}$. After washing the neurons were triturated, counted and plated in Neurobasal (E18 neurons) medium (Invitrogen, Carlsbad, USA) supplemented with 2\% B-27 (Invitrogen), 1.8\% HEPES, $1 \%$ glutamax (Invitrogen), $1 \%$ Pen/Strep (Invitrogen) and $0.2 \% \beta$-mercaptoethanol. Neurons were plated at 2,500 cells $\mathrm{cm}^{-2}$ on mouse glia microislands. Glial islands were prepared by first coating glass coverslips with $0.15 \%$ agarose. After drying and ultraviolet sterilization, custom-made rubber stamps were used to print dots (islands, diameter $200-250 \mu \mathrm{m}$ ) using a substrate mixture containing $0.25 \mathrm{mg} \mathrm{ml}^{-1}$ rat tail collagen and $0.4 \mathrm{mg} \mathrm{ml}^{-1}$ poly-D-lysine dissolved in $17 \mathrm{mM}$ acetic acid; glial cells were plated at 4,800 cells $\mathrm{cm}^{-2}$. Neurons were transduced (DIV 5) with bicistronic AAV virus co-expressing Tau and GFP (to indicate transduced neurons), or transduced with control AAV virus expressing GFP alone. For competition experiments, transduced neurons expressing Tau_P301L were pretreated with $5 \mu \mathrm{M}$ Tat-NT ${ }^{\text {Tau }}$ or Tat-mCherry peptide for four hours before recording.

Electrophysiological recordings of rat neurons. Cultured hippocampal neurons from Wistar rat embryos were recorded on DIV 11-13. The intracellular pipette solution contained (in $\mathrm{mM}$ ): $136 \mathrm{KCl}, 18$ HEPES, $4 \mathrm{Na}$-ATP, $4.6 \mathrm{MgCl}_{2}$, $4 \mathrm{~K}_{2}$-ATP, 15 Creatine Phosphate, 1 EGTA and $50 \mathrm{U} / \mathrm{ml}$ Phospocreatine Kinase $(300 \mathrm{mOsm}$, $\mathrm{pH}$ 7.30). The extracellular solution used during recordings contained the following components (in $\mathrm{mM}$ ): $140 \mathrm{NaCl}, 2.4 \mathrm{KCl}, 4 \mathrm{CaCl}_{2}, 4 \mathrm{MgCl}_{2}, 10$ HEPES, 10 Glucose (300 mOsm, $\mathrm{pH} 7.30)$. Only islands with singular neurons were recorded (whole-cell voltage clamped at $-70 \mathrm{mV}$ ) using a double EPC-10 amplifier (HEKA Elektronik, Lambrecht/Pfalz, Germany) under control of Patchmaster v2 $\times 32$ software (HEKA Elektronik). Currents were recorded at $20 \mathrm{~Hz}$ and low-pass filtered at $3 \mathrm{kHz}$ when stored. Pipettes were pulled using a Sutter P-1000 and resistance ranged from 3 to $5 \mathrm{M} \Omega$. The series resistance was compensated to 75 $85 \%$. Cells with series resistances above $15 \mathrm{M} \Omega$ were excluded for analysis. All recordings were performed at room temperature. Spontaneous glutamatergic release (mEPSCs) was recorded at $-70 \mathrm{mV}$. Evoked release was induced using brief depolarizatingof the cell soma (from -70 to $0 \mathrm{mV}$ for $1 \mathrm{~ms}$ ) to initiate action potential dependent glutamatergic release (eEPSCs).

Adeno-associated viral vectors. The complementary DNA (cDNA) of HA tagged wild type or mutant human Tau (0N4R) was subcloned into a bicistronic adeno-associated viral serotype 6 (AAV6) vector containing eGFP cDNA. The co-expression of eGFP and HA-Tau was driven by a human synapsin-1 promoter (Genscript, Piscataway, NJ, USA). Recombinant AAV vectors were propagated in HEK293 cells, purified by iodixanol step gradient ultracentrifugation and heparin affinity FPLC, followed by extensive dialysis against PBS. Genome copies were 
determined by quantitative real time PCR and purity $>99 \%$ by SDS gel electrophoresis and silver staining.

Array tomography of human brain tissue. Human brain samples from superior temporal gyrus were provided by collaborating neuropathologists Prof. Colin Smith (University of Edinburgh Sudden Death Brain Bank) and Prof Matthew Frosch (Massachusetts ADRC brain bank). Use of human tissue followed national and institutional ethics guidelines, and was approved by the Edinburgh Brain Bank Ethics Committee and the Academic and Clinical Central Office for Research and Development medical research ethics committee. Brain tissue was collected at autopsy, small tissue blocks of approximately $1 \times 1 \times 5 \mathrm{~mm}^{3}$ containing all six cortical layers from the pia to white matter were cut with a razor blade, and samples were fixed in $4 \%$ paraformaldehyde with $2.5 \%$ sucrose for $2-3 \mathrm{~h}$. Tissue was then dehydrated and embedded in LR white resin. Embedded blocs were cut with an ultramicrotome (Leica Ultracut) into ribbons of serial ultrathin $(70 \mathrm{~nm})$ sections, which were mounted on gelatin subbed coverslips (Fisher \#1.5). Sections were blocked in $0.05 \%$ Tween and $0.1 \%$ BSA in TBS for 30 min followed by overnight incubation with primary antibodies diluted in blocking buffer: Synapsin I (1:100, rabbit, Millipore AB1543) and AT8 (1:50, mouse, Thermo Scientific MN1020). The next day, sections were stained with for $30 \mathrm{~min}$ in the following secondary antibody solutions diluted 1:50 in blocking buffer: Alexa594-conjugated donkey anti rabbit antibodies (Invitrogen A21207) and Alexa488-conjugated donkey anti mouse antibodies (Invitrogen A21202). DAPI $\left(0.005 \mathrm{mg} \mathrm{ml}^{-1}\right)$ was included in the secondary antibody solution to stain nuclei. Images were acquired on a Zeiss axio Imager Z2 epifluorescence scope. A tilescan image was acquired with a $\times 10$ objective of DAPI staining on the entire ribbon. High resolution images were acquired in the same location on every section on the ribbon using a $\times 63$ oil 1.4 NA objective. Same exposure times were used to capture all images (chosen on an $\mathrm{AD}$ case with tau pathology).

Immunoblotting of Drosophila and human brain samples. To compare the expression of human Tau protein in fly heads, UAS-human Tau fly lines from the current work (UAS-Tau ${ }^{\text {WT }} 68$ A, UAS-Tau ${ }^{\text {R406W }} 68$ A, UAS-Tau V337M 68 A and UAS-Tau ${ }^{P 301 L}{ }_{68}^{6}$ ) and from the previous work (UAS-Tau ${ }^{\text {WT }}{ }_{-} \mathrm{H}$ and UAS$\left.\mathrm{Tau}^{\mathrm{R} 406 \mathrm{~W}}{ }_{-} \mathrm{H}\right)^{35-38}$ were crossed to pan-neuronal driver Elav-Gal4 and maintained at $25^{\circ} \mathrm{C}$. The offspring from were collected on the day of eclosion and the fly heads were homogenized in RIPA buffer ( $10 \mathrm{mM}$ Tris-Cl pH 8.0, $1 \mathrm{mM}$ EDTA, $0.5 \mathrm{mM}$ EGTA, $1 \%$ Triton X-100, $1 \%$ SDS, $140 \mathrm{mM} \mathrm{NaCl}$, complete protease inhibitor). Lysates were incubated on ice for $15 \mathrm{~min}$ and centrifuged at 14,000 r.p.m. for $15 \mathrm{~min}$ at $4{ }^{\circ} \mathrm{C}$. Protein concentration was determined using the BCA protein assay (Thermo Scientific). Samples were separated by SDS-polyacrylamide gel electrophoresis (SDS-PAGE) and analysed by immunoblotting (described above) for total Tau (DAKO, A0024, 1:1,000) and Tubulin (DSHB, E7, 1:5,000). The expression of human Tau protein in fly heads is further compared to that in human brains. Control human autopsy brain tissue from entorhinal cortex region was obtained from London Neurodegenerative Disease Brain Bank. Human brain lysates were prepared by homogenization in 2\% SDS by Fastprep Machine on a program of $45 \mathrm{~s}-6000$ shakes per $\mathrm{s}$ and cleared by centrifugation at 14,000 r.p.m. for $15 \mathrm{~min}$ at $4{ }^{\circ} \mathrm{C}$. Protein concentration was determined using BCA protein assay (Thermo Scientific). Equal amount of total proteins $(15 \mu \mathrm{g})$ from both fly brains and human brains were separated by SDS-PAGE and analysed by immunoblotting (above) for total Tau (DAKO, A0024, 1:1,000) and Tubulin (DSHB, E7, 1:5,000). The relative Tau expression levels were quantified based on the intensities of protein bands from the immunoblots as the data represents the Tau protein levels from equal amounts of total protein from fly heads and human brains used for loading on SDS-PAGE.

Isolation of synaptic vesicles from mouse brain. All animal experiments were performed with ethical permission from and under the guidelines of the $\mathrm{KU}$ Leuven animal ethics committee. To prepare synaptic vesicles, brains of six-week old wild type mice were isolated, pooled and homogenized in ice-cold homogenization buffer ( $320 \mathrm{mM}$ sucrose, $4 \mathrm{mM}$ HEPES pH 7.4, Complete protease inhibitors; $2 \mathrm{ml}$ buffer per brain) with a Potter-Elvehjem Teflon glass homogenizer by 10 strokes at 600 r.p.m. Homogenates were centrifuged at $800 \mathrm{~g}$ for $10 \mathrm{~min}$ to remove nucleus and intact cells, and the supernatants were recovered and further centrifuged at $10,000 \mathrm{~g}$ for $15 \mathrm{~min}$. The pellet was washed once with homogenization buffer and collected again by centrifugation at $10,000 \mathrm{~g}$ for $15 \mathrm{~min}$. The washed pellet fraction $\left(\mathrm{P}^{\prime}\right)$ was then resuspended in homogenization buffer (100 $\mu \mathrm{l}$ per each hemisphere), and mixed with 9 volumes of ice-cold water (supplemented with $5 \mathrm{mM}$ HEPES-KOH, $\mathrm{pH} 7.4$ and protease inhibitors) to induce osmotic shock with mixing during rotation for $30 \min 4{ }^{\circ} \mathrm{C}$ and then subjected to centrifugation at $25,000 \mathrm{~g}$ for $20 \mathrm{~min}$. The pellet fraction (LP1) was discarded, and the supernatant (LS1) was further centrifuged at $200,000 \mathrm{~g}$ for $2 \mathrm{~h}$ to collect the crude synaptic vesicle fraction (LP2), which was resuspended in $5 \mathrm{mM}$ HEPES $\mathrm{pH}$ $7.4,300 \mathrm{mM}$ glycine. All manipulation and centrifugation procedures were performed at $4^{\circ} \mathrm{C}$, and buffers were supplemented with fresh Complete protease inhibitor cocktail.

To check vesicle enrichment, fractions collected during the vesicle preparation were lysed in RIPA buffer (Sigma) with protease inhibitors. Protein concentrations were measured and equal amounts of protein were reduced in LDS sample buffer and probed for presynaptic and postsynaptic membrane markers by immunoblotting.

Purification of recombinant human Tau. The cDNAs encoding full-length or domain-truncated human Tau (0N4R isoform) were cloned into the BamHI and EcoRI restriction sites of pGEX-6P-1 (GE Healthcare) using a reverse primer encoding a $8 \mathrm{x}$-His tag. The resulting fusion proteins contained an N-terminal GST tag followed by a PreScission Protease cleavage site, Tau and a C-terminal His tag. The plasmid was transformed into Rosetta bacteria (Merck Millipore) for recombinant expression. For expression, bacteria were inoculated in LB medium containing ampicillin and chloramphenicol and grown overnight at $37^{\circ} \mathrm{C}$. The next day, bacteria were diluted to an $\mathrm{OD}_{600}$ of 0.2 , and let grow at $37^{\circ} \mathrm{C}$ until the culture reached an $\mathrm{OD}_{600}$ of 1.0 , at which point IPTG (Thermo Scientific) was added to a final concentration of $0.4 \mathrm{mM}$ to induce recombinant protein expression. Bacteria were incubated for $2 \mathrm{~h}$ at $37^{\circ} \mathrm{C}$ following induction, then were immediately pelleted and stored at $-80^{\circ} \mathrm{C}$ until lysis. For cell lysis, frozen bacterial cell pellets were resuspended in bacterial lysis buffer (PBS supplemented with 10\% glycerol, $1 \%$ Triton-X-100, $1 \mathrm{mM}$ PMSF, Complete protease inhibitors (Roche), lysozyme and Benzonase (Sigma) and incubated at $4^{\circ} \mathrm{C}$ for $30 \mathrm{~min}$ with rotation, followed by centrifugation at $16,000 \mathrm{~g}$ for $20 \mathrm{~min}$. Cleared cell lysates were loaded onto prewashed Glutathione Sepharose 4B (GE Healthcare) and incubated for 2 hours at $4^{\circ} \mathrm{C}$ with gentle rotation. After the incubation, the resin was washed twice with PBS supplemented with $250 \mathrm{mM} \mathrm{NaCl}$, and then twice with PreScission Protease cleavage buffer (20 mM Tris- $\mathrm{HCl} \mathrm{pH} \mathrm{7.0,50} \mathrm{mM} \mathrm{NaCl,} 0.5 \mathrm{mM}$ EDTA, $1 \mathrm{mM}$ DTT, $0.01 \%$ Tween-20). Proteolytic cleavage of the N-terminal GST tag was performed by incubation with PreScission Protease (GE Healthcare) overnight at $4{ }^{\circ} \mathrm{C}$. The following day, the supernatant of the cleavage reaction was collected and incubated with fresh Glutathione Sepharose for $1 \mathrm{~h}$ at $4{ }^{\circ} \mathrm{C}$ to remove residual GSTtagged protease and uncleaved protein. Proteins were further purified against the C-terminal His tag by applying the supernatant onto washed Ni-NTA Profinity Resin (BioRad) and bound for $45 \mathrm{~min}$ at $4{ }^{\circ} \mathrm{C}$. The Ni-NTA resin was subsequently washed three times in buffer containing $50 \mathrm{mM} \mathrm{NaH}_{2} \mathrm{PO}_{4} \mathrm{pH} 8.0,300 \mathrm{mM} \mathrm{NaCl}$, $20 \mathrm{mM}$ imidazole before eluting bound His-tagged buffer in the same buffer containing $250 \mathrm{mM}$ imidazole. Protein eluates were concentrated using $0.5 \mathrm{ml}$ centrifugal filter units (Merck Millipore) with a $10 \mathrm{kDa}$ MWCO. Protein was quantified using Quick Start Bradford Reagent (BioRad). Protein purity was evaluated with Page Blue colloidal coomassie staining solution (Thermo Scientific). All in vitro binding experiments utilized freshly purified Tau protein immediately following purification.

Co-Immunoprecipitation of Tau and synaptic vesicles. In this co-IP assay, recombinant Tau-His was used as bait to pull-down intact synaptic vesicles prepared from mouse brains. Briefly, $1 \mu \mathrm{g}$ of freshly purified recombinant Tau-His protein was immobilized to Protein G Magnetic Dynabeads (Invitrogen) using anti-His antibodies (Invitrogen, clone 4A12E4) in vesicle binding buffer supplemented with $1 \% \mathrm{BSA}$ for $2 \mathrm{~h}$ at $4{ }^{\circ} \mathrm{C}$. The Tau-bound Dynabeads were then washed and incubated with mouse crude synaptic vesicles (LP2 fraction, equivalent to $20 \mu \mathrm{g}$ of protein) in synaptic vesicle binding buffer supplemented with $1 \%$ BSA, $0.1 \%$ NP$40,0.1 \%$ Tween-20, and protease inhibitors in a $500 \mu \mathrm{l}$ reaction mixture for $2 \mathrm{~h}$ at $4^{\circ} \mathrm{C}$. Note that this low-concentration of detergents increases specificity of binding without lysing synaptic vesicles. The Dynabeads were thoroughly washed after incubation, and the bound proteins were retrieved by boiling in LDS containing $1 \% \beta$-mercaptoethanol and assessed for the presence of synaptic vesicle marker proteins by immunoblotting. For the competition experiment with full-length Tau and Tat-NT Tau, intact synaptic vesicles (equivalent to $20 \mu \mathrm{g}$ of protein) were immobilized on Dynabeads using $1 \mu \mathrm{g}$ anti-Synaptobrevin 2 antibodies (Synaptic Systems, clone 69.1) for $4 \mathrm{~h}$ at $4{ }^{\circ} \mathrm{C}$. After 4 hours, SV-bound Dynabeads were washed and then resuspended in vesicle binding buffer containing $1 \mu \mathrm{g}$ $(40 \mathrm{nM})$ of freshly purified full-length Tau-His with varying concentrations of freshly purified Tat-NT Tau - His. Reactions were left mixing at $4^{\circ} \mathrm{C}$ for $2 \mathrm{~h}$, after which beads were thoroughly washed and then boiled in LDS and assessed for the amount of full-length Tau bound to synaptic vesicles by immunoblotting.

EM analysis of Tau-vesicle binding in vitro. To assess the binding of recombinant Tau-His to ultrapure rat synaptic vesicles, $1 \mu \mathrm{M}$ Tau-His and $50 \mu \mathrm{g} \mathrm{ml}^{-1}$ vesicles were incubated together in synaptic vesicle binding buffer containing $1 \%$ BSA for $2 \mathrm{~h}$ at $4{ }^{\circ} \mathrm{C}$ in a $100 \mu \mathrm{l}$ binding reaction. After binding, $2 \mu \mathrm{l}$ of the vesicle suspension was dotted onto a glow-discharged, carbon-coated grid and let air dry. After drying, grids were blocked in 1\% BSA for 5 min followed by incubation with $20 \mathrm{nM}$ of $5 \mathrm{~nm}$ Ni-NTA-Nanogold (Nanoprobes) for $30 \mathrm{~min}$ in SV buffer containing $1 \%$ BSA. Grids were subsequently washed in SV buffer containing $8 \mathrm{mM}$ imidazole before fixation in $2 \%$ glutaraldehyde for $5 \mathrm{~min}$. Grids were then washed in ultrapure water before negative staining with $1 \%$ uranyl acetate. Samples were imaged on a Jeol JEM1400 transmission electron microscope.

In vitro binding of phospho-Tau to synaptic vesicles. Recombinant human Tau (2N4R isoform, 44laa) was purified from bacterial culture (Tebu-Bio) and introduced into an in vitro phosphorylation reaction with human GSK3- $\beta$ and CDK5 kinases purified from insect cells (Merck Millipore) in buffer containing $40 \mathrm{mM}$ 
Tris- $\mathrm{HCl}$ (pH 7.2), $150 \mathrm{mM} \mathrm{NaCl}, 0.2 \%$ n-octyl-glucoside, $5 \mathrm{mM} \mathrm{MgCl}, 1 \mathrm{mM}$ EDTA, $2 \%$ glycerol, $5 \mathrm{mM}$ ATP, $0.25 \mathrm{mg} \mathrm{ml}^{-1}$ Ovalbumin (GE Healthcare), $1 \mathrm{mM}$ DTT and $0.1 \mathrm{mM} \mathrm{Na}_{2} \mathrm{VO}_{4}$. The reaction was allowed to proceed for $7 \mathrm{~h}$ at $35^{\circ}$. After $7 \mathrm{~h}$, pure phosphorylated Tau was separated away from kinases and Tau degradation products by size exclusion chromatography on a Superdex-200 column. pTau-containing fractions were pooled, concentrated and stored at $-80^{\circ}$ for later use. Equal purity of Tau and pTau was confirmed by colloidal coomassie staining. To test the synaptic vesicle binding affinity of pTau, we thawed aliquots of Tau and pTau and pre-cleared aggregates at 100,000 $g$ for $1 \mathrm{~h}$. The supernatant was collected, protein concentration determined, and $500 \mathrm{ng}$ of Tau or pTau was introduced into the sedimentation assay with synaptic vesicles prepared from mouse brains. Tau bound to vesicles was detected by immunoblotting the synaptic vesicle pellet with anti-total Tau (DAKO, 1:1000) antibodies.

Immunofluorescence labelling of rat hippocampal neurons. Rat autaptic hippocampal neuronal cultures were transduced with AAV expressing Tau variants. At DIV 11, the cells were fixed in $4 \%$ paraformaldehyde $+4 \%$ sucrose in PBS for $25 \mathrm{~min}$ at room temperature. After fixation, coverslips were washed for three times with PBS and incubated with blocking buffer (PBS containing $5 \%$ goat serum, $3 \% \mathrm{BSA}$ and $0.3 \%$ Triton-X) for $1 \mathrm{~h}$ at room temperature. The coverslips were then incubated with primary antibodies overnight at $4{ }^{\circ} \mathrm{C}$. Both primary and secondary antibodies were diluted in buffer PBS containing $1 \%$ goat serum, $2 \%$ BSA and $0.1 \%$ Triton-X. The next day, the coverslips were washed for three times with PBS and then incubated with secondary antibodies for $1 \mathrm{~h}$ at room temperature. After washing for five times with PBS, the immunolabled coverslips were mounted on glass slides with Vectashield mounting medium (Vector laboratories). The following antibodies were used: mouse anti-HA (Covance; 1:500), rabbit antiSynapsin (Merck Millipore; 1:500) and chicken anti-MAP2 (abcam; 1:5000), Alexa555 goat-anti-mouse, Alex-647 goat-anti-chicken and Pacific Blue goat-anti-rabbit (Invitrogen; 1:5000). All digital images were captured using a Nikon confocal microscope with a $\times 60$ oil lens, and were further processed using Image $\mathrm{J}$ software. For quantification of the images, rectangles with area of $\sim 2,500 \mu \mathrm{m}^{2}$ containing distal axons were randomly selected, and the numbers of Tau/Synapsin dual-positive puncta and Synapsin-positive puncta within the selected areas were counted manually.

Histology of fly brains. Adult fly heads were fixed in PBS containing $4 \%$ formalin for $4 \mathrm{~h}$ at room temperature. After fixation, samples were dehydrated in ethanol series and embedded in paraffin overnight. A microtome (Leica RM2135) with a disposable knife was used to make frontal sections of the adult heads. A series of sections $(5 \mu \mathrm{m})$ spanning the brain were collected on a glass slide and subject to H\&E histology staining according to standard protocols. Stained sections were visualized using a microscope (LeicaDMRA2), and images were acquired with a digital camera (Photometrics, CoolSnap 5.0) steered by the Northern Eclipse 6.0 software (EMPIX Imaging Inc.).

Phalloidin staining of hippocampal neurons. Primary hippocampal neurons were isolated (described above) and plated at density of 140 cells $\mathrm{mm}^{-2}$ on poly-Llysine coated coverslips. Neurons were transduced (at DIV 5) with bicistronic AAV virus co-expressing Tau and GFP (to indicate transduced neurons), or transduced with control AAV virus expressing GFP alone. At DIV 11, transduced neurons expressing Tau_P301L were pretreated with $5 \mu \mathrm{M}$ Tat-NT Tau for $4 \mathrm{~h}$. Neurons were fixed in $4 \%$ paraformaldehyde, $4 \%$ sucrose in PBS and blocked with blocking solution (5\% goat serum, 3\% BSA, $0.3 \%$ Triton-X-100 in PBS). The coverslips were incubated with rabbit anti-Synaptophysin I antibody (Synaptic Systems, 1:1,000) in incubation buffer ( $1 \%$ goat serum, $2 \%$ BSA, $0.1 \%$ Triton-X-100 in PBS) overnight at $4{ }^{\circ} \mathrm{C}$. The next day, the coverslips were washed and incubated with antirabbit Pacific Blue secondary antibodies (Life Technologies, 1: 5,000) and $1 \mu \mathrm{g} \mathrm{ml}^{-1}$ TRITC-Phalloidin (Sigma) for $1 \mathrm{~h}$ at room temperature, followed by washing and mounting. Synaptophysin-labelled punctae along the axon were used to indicate presynaptic compartments. The amount of presynaptic F-actin was quantified by measuring the Phalloidin fluorescence intensity (integrated density) within hand-drawn ROIs around Synaptophysin-labelled punctae using ImageJ.

Statistics. The results in graphs depicted in dot plots are shown as average \pm s.e.m. unless otherwise noted. Statistical testing was performed using GraphPad and SigmaPlot 12.3 (Systat Software Inc), Student $t$-test, One-Way ANOVA and TwoWay ANOVA. The significance levels are indicated by asterisks; ${ }^{*} P<0.05$; ${ }^{*} P<0.01 ;{ }^{* *} P<0.001$.

Data availability. The data that support the current findings are available from the corresponding author on request.

\section{References}

1. Morris, M., Maeda, S., Vossel, K. \& Mucke, L. The many faces of tau. Neuron 70, 410-426 (2011).
2. Ballatore, C., Lee, V. M. \& Trojanowski, J. Q. Tau-mediated neurodegeneration in Alzheimer's disease and related disorders. Nat. Rev. Neurosci. 8, 663-672 (2007).

3. Wang, Y. \& Mandelkow, E. Tau in physiology and pathology. Nat. Rev. Neurosci. 17, 22-35 (2016).

4. Kwok, J. B. et al. Tau haplotypes regulate transcription and are associated with Parkinson's disease. Ann. Neurol. 55, 329-334 (2004).

5. Refenes, N. et al. Role of the H1 haplotype of microtubule-associated protein tau (MAPT) gene in Greek patients with Parkinson's disease. BMC Neurol. 9, 26 (2009).

6. Allen, M. et al. Association of MAPT haplotypes with Alzheimer's disease risk and MAPT brain gene expression levels. Alzheimers Res. Ther. 6, 39 (2014).

7. Liu, T. et al. Amyloid-beta-induced toxicity of primary neurons is dependent upon differentiation-associated increases in tau and cyclin-dependent kinase 5 expression. J. Neurochem. 88, 554-563 (2004).

8. Rapoport, M., Dawson, H. N., Binder, L. I., Vitek, M. P. \& Ferreira, A. Tau is essential to beta -amyloid-induced neurotoxicity. Proc. Natl Acad. Sci. USA 99, 6364-6369 (2002).

9. Nussbaum, J. M. et al. Prion-like behaviour and tau-dependent cytotoxicity of pyroglutamylated amyloid- $\beta$. Nature 485, 651-655 (2012).

10. Roberson, E. D. et al. Reducing endogenous tau ameliorates amyloid betainduced deficits in an Alzheimer's disease mouse model. Science 316, 750-754 (2007).

11. Holth, J. K. et al. Tau loss attenuates neuronal network hyperexcitability in mouse and Drosophila genetic models of epilepsy. J. Neurosci. 33, 1651-1659 (2013).

12. DeVos, S. L. et al. Antisense reduction of tau in adult mice protects against seizures. J. Neurosci. 33, 12887-12897 (2013).

13. Masliah, E. et al. Altered expression of synaptic proteins occurs early during progression of Alzheimer's disease. Neurology 56, 127-129 (2001).

14. Bellucci, A., Navarria, L., Zaltieri, M., Missale, C. \& Spano, P. $\alpha$-Synuclein synaptic pathology and its implications in the development of novel therapeutic approaches to cure Parkinson's disease. Brain Res. 1432, 95-113 (2012).

15. Milnerwood, A. J. \& Raymond, L. A. Early synaptic pathophysiology in neurodegeneration: insights from Huntington's disease. Trends Neurosci. 33, 513-523 (2010).

16. Scheff, S. W., Price, D. A., Schmitt, F. A. \& Mufson, E. J. Hippocampal synaptic loss in early Alzheimer's disease and mild cognitive impairment. Neurobiol. Aging 27, 1372-1384 (2006).

17. Yoshiyama, Y. et al. Synapse loss and microglial activation precede tangles in a P301S tauopathy mouse model. Neuron 53, 337-351 (2007).

18. Crimins, J. L., Rocher, A. B. \& Luebke, J. I. Electrophysiological changes precede morphological changes to frontal cortical pyramidal neurons in the rTg4510 mouse model of progressive tauopathy. Acta Neuropathol. 124, 777-795 (2012).

19. Polydoro, M. et al. Soluble pathological tau in the entorhinal cortex leads to presynaptic deficits in an early Alzheimer's disease model. Acta Neuropathol. 127, 257-270 (2014).

20. Sydow, A. et al. Tau-induced defects in synaptic plasticity, learning, and memory are reversible in transgenic mice after switching off the toxic Tau mutant. J. Neurosci. 31, 2511-2525 (2011).

21. Hoover, B. R. et al. Tau mislocalization to dendritic spines mediates synaptic dysfunction independently of neurodegeneration. Neuron 68, 1067-1081 (2010).

22. Erez, H., Shemesh, O. A. \& Spira, M. E. Rescue of tau-induced synaptic transmission pathology by paclitaxel. Front. Cell Neurosci. 8, 34 (2014).

23. Menkes-Caspi, N. et al. Pathological tau disrupts ongoing network activity. Neuron 85, 959-966 (2015).

24. Santacruz, K. et al. Tau suppression in a neurodegenerative mouse model improves memory function. Science 309, 476-481 (2005).

25. Hong, M. et al. Mutation-specific functional impairments in distinct tau isoforms of hereditary FTDP-17. Science 282, 1914-1917 (1998).

26. Fein, J. A. et al. Co-localization of amyloid beta and tau pathology in Alzheimer's disease synaptosomes. Am. J. Pathol. 172, 1683-1692 (2008).

27. Sokolow, S. et al. Pre-synaptic C-terminal truncated tau is released from cortical synapses in Alzheimer's disease. J. Neurochem. 133, 368-379 (2015).

28. Henkins, K. M. et al. Extensive p-tau pathology and SDS-stable p-tau oligomers in Alzheimer's cortical synapses. Brain Pathol. 22, 826-833 (2012).

29. Tai, H. C. et al. The synaptic accumulation of hyperphosphorylated tau oligomers in Alzheimer disease is associated with dysfunction of the ubiquitinproteasome system. Am. J. Pathol. 181, 1426-1435 (2012).

30. Sahara, N., Murayama, M., Higuchi, M., Suhara, T. \& Takashima, A. Biochemical distribution of Tau protein in synaptosomal fraction of transgenic mice expressing human P301L Tau. Front. Neurol. 5, 26 (2014).

31. Harris, J. A. et al. Human P301L-mutant tau expression in mouse entorhinalhippocampal network causes tau aggregation and presynaptic pathology but no cognitive deficits. PLoS ONE 7, e45881 (2012). 
32. Ittner, L. M. et al. Dendritic function of tau mediates amyloid-beta toxicity in Alzheimer's disease mouse models. Cell 142, 387-397 (2010).

33. Lloyd, T. E. et al. A genome-wide search for synaptic vesicle cycle proteins in Drosophila. Neuron 26, 45-50 (2000)

34. Hadley, D. et al. Patterns of sequence conservation in presynaptic neural genes. Genome Biol. 7, R105 (2006).

35. Dias-Santagata, D., Fulga, T. A., Duttaroy, A. \& Feany, M. B. Oxidative stress mediates tau-induced neurodegeneration in Drosophila. J. Clin. Invest. 117, 236-245 (2007)

36. Frost, B., Hemberg, M., Lewis, J. \& Feany, M. B. Tau promotes neurodegeneration through global chromatin relaxation. Nat. Neurosci. 17, 357-366 (2014).

37. Khurana, V. et al. TOR-mediated cell-cycle activation causes neurodegeneration in a Drosophila tauopathy model. Curr. Biol. 16, 230-241 (2006).

38. Wittmann, C. W. et al. Tauopathy in Drosophila: neurodegeneration without neurofibrillary tangles. Science 293, 711-714 (2001).

39. Kawasaki, F., Hazen, M. \& Ordway, R. W. Fast synaptic fatigue in shibire mutants reveals a rapid requirement for dynamin in synaptic vesicle membrane trafficking. Nat. Neurosci. 3, 859-860 (2000).

40. Verstreken, P. et al. Endophilin mutations block clathrin-mediated endocytosis but not neurotransmitter release. Cell 109, 101-112 (2002).

41. Saheki, Y. \& De Camilli, P. Synaptic vesicle endocytosis. Cold Spring Harb. Perspect. Biol. 4, a005645 (2012)

42. Sara, Y., Mozhayeva, M. G., Liu, X. \& Kavalali, E. T. Fast vesicle recycling supports neurotransmission during sustained stimulation at hippocampal synapses. J. Neurosci. 22, 1608-1617 (2002).

43. Verstreken, P. et al. Synaptic mitochondria are critical for mobilization of reserve pool vesicles at Drosophila neuromuscular junctions. Neuron 47, 365-378 (2005)

44. Alabi, A. A. \& Tsien, R. W. Synaptic vesicle pools and dynamics. Cold Spring Harb. Perspect. Biol. 4, a013680 (2012).

45. Fernández-Alfonso, T. \& Ryan, T. A. The kinetics of synaptic vesicle pool depletion at CNS synaptic terminals. Neuron 41, 943-953 (2004).

46. Gaffield, M. A., Rizzoli, S. O. \& Betz, W. J. Mobility of synaptic vesicles in different pools in resting and stimulated frog motor nerve terminals. Neuron 51, 317-325 (2006)

47. Miesenböck, G., De Angelis, D. A. \& Rothman, J. E. Visualizing secretion and synaptic transmission with $\mathrm{pH}$-sensitive green fluorescent proteins. Nature 394, 192-195 (1998)

48. Fulga, T. A. et al. Abnormal bundling and accumulation of F-actin mediates tau-induced neuronal degeneration in vivo. Nat. Cell Biol. 9, 139-148 (2007).

49. He, H. J. et al. The proline-rich domain of tau plays a role in interactions with actin. BMC Cell Biol. 10, 81 (2009).

50. Shupliakov, O., Haucke, V. \& Pechstein, A. How synapsin I may cluster synaptic vesicles. Semin. Cell Dev. Biol. 22, 393-399 (2011).

51. Steinhilb, M. L., Dias-Santagata, D., Fulga, T. A., Felch, D. L. \& Feany, M. B. Tau phosphorylation sites work in concert to promote neurotoxicity in vivo. Mol. Biol. Cell 18, 5060-5068 (2007).

52. Bekkers, J. M. \& Stevens, C. F. Excitatory and inhibitory autaptic currents in isolated hippocampal neurons maintained in cell culture. Proc. Natl Acad. Sci. USA 88, 7834-7838 (1991).

53. Chouhan, A. K. et al. Uncoupling neuronal death and dysfunction in Drosophila models of neurodegenerative disease. Acta Neuropathol. Commun. 4, 62 (2016).

54. Diao, J. et al. Native $\alpha$-synuclein induces clustering of synaptic-vesicle mimics via binding to phospholipids and synaptobrevin-2/VAMP2. Elife 2, e00592 (2013).

55 . Wang, L. et al. $\alpha$-synuclein multimers cluster synaptic vesicles and attenuate recycling. Curr. Biol. 24, 2319-2326 (2014).

56. Fioravante, D., Liu, R. Y., Netek, A. K., Cleary, L. J. \& Byrne, J. H. Synapsin regulates Basal synaptic strength, synaptic depression, and serotonin-induced facilitation of sensorimotor synapses in Aplysia. J. Neurophysiol. 98, 3568-3580 (2007).

57. Vasileva, M., Renden, R., Horstmann, H., Gitler, D. \& Kuner, T. Overexpression of synapsin Ia in the rat calyx of Held accelerates short-term plasticity and decreases synaptic vesicle volume and active zone area. Front. Cell Neurosci. 7, 270 (2013)

58. Zhao, X. et al. Caspase-2 cleavage of tau reversibly impairs memory. Nat. Med. 22, 1268-1276 (2016).

59. Penzes, P. et al. Rapid induction of dendritic spine morphogenesis by transsynaptic ephrinB-EphB receptor activation of the Rho-GEF kalirin. Neuron 37, 263-274 (2003)

60. Rao, A., Cha, E. M. \& Craig, A. M. Mismatched appositions of presynaptic and postsynaptic components in isolated hippocampal neurons. J. Neurosci. 20 8344-8353 (2000)
61. Crimins, J. L. et al. Homeostatic responses by surviving cortical pyramidal cells in neurodegenerative tauopathy. Acta Neuropathol. 122, 551-564 (2011).

62. Decker, J. M. et al. Pro-aggregant Tau impairs mossy fiber plasticity due to structural changes and $\mathrm{Ca}(++)$ dysregulation. Acta Neuropathol. Commun. 3 , 23 (2015).

63. Rocher, A. B. et al. Structural and functional changes in tau mutant mice neurons are not linked to the presence of NFTs. Exp. Neurol. 223, 385-393 (2010).

64. Crone, N. E., Korzeniewska, A. \& Franaszczuk, P. J. Cortical $\gamma$ responses: searching high and low. Int. J. Psychophysiol. 79, 9-15 (2011).

65. Herrmann, C. S., Munk, M. H. \& Engel, A. K. Cognitive functions of gammaband activity: memory match and utilization. Trends Cogn. Sci. 8, 347-355 (2004).

66. Lachaux, J. P., Axmacher, N., Mormann, F., Halgren, E. \& Crone, N. E. High frequency neural activity and human cognition: past, present and possible future of intracranial EEG research. Prog Neurobiol. 98, 279-301 (2012).

67. Ferreira, A. \& Bigio, E. H. Calpain-mediated tau cleavage: a mechanism leading to neurodegeneration shared by multiple tauopathies. Mol. Med. 17, 676-685 (2011).

68. Vilain, S. et al. Fast and efficient Drosophila melanogaster gene knock-ins using MiMIC transposons. G3 4, 2381-2387 (2014).

69. Uytterhoeven, V., Kuenen, S., Kasprowicz, J., Miskiewicz, K. \& Verstreken, P. Loss of skywalker reveals synaptic endosomes as sorting stations for synaptic vesicle proteins. Cell 145, 117-132 (2011).

70. Holt, M., Riedel, D., Stein, A., Schuette, C. \& Jahn, R. Synaptic vesicles are constitutively active fusion machines that function independently of $\mathrm{Ca}^{2}+$ Curr. Biol. 18, 715-722 (2008).

\section{Acknowledgements}

We thank Colin Smith and Matthew Frosch for access to human tissue. We thank lab members of the Verstreken laboratory for helpful discussions, and Kristel Vennekens and An Snellinx for technical support. Support was provided by an ERC Starting Grant (260678), ERC Consolidator grant (646671), the Instituut voor Wetenschap en Technologie (IWT O\&O grant), the Interuniversity Attraction Pole program by BELSPO, the research fund KU Leuven, a Methusalem grant of the Flemish government and VIB, Leuvens Universiteitsfonds (LUF) Opening the Future grant, and a Belgian-American Educational Foundation fellowship to J.M. T.S.-J., A.H. and R.J.J. receive funding from Alzheimer's Research UK, an anonymous foundation, and a Welcome Trust Institutional strategic support grant.

\section{Author contributions}

L.Z., J.M. and P.V. conceived the study and wrote the manuscript. L.Z., J.M. and K.W. conducted the experiments. A.G.H., R.J.J. and T.L.S.-J. performed array tomography analysis. Y.W. performed histology analysis of fly brain, J.S. and K.M. performed EM analysis. M.H. prepared ultrapure synaptic vesicles, J.B. provided technical support and S.V. helped with fly genetics. M.H., I.D., D.M., J.D.W., and B.D.S. contributed to the design of the study. B.D.S. contributed to the writing of the manuscript and all other co-authors edited the manuscript.

\section{Additional information}

Supplementary Information accompanies this paper at http://www.nature.com/ naturecommunication

Competing interests: The authors declare no competing financial interests.

Reprints and permission information is available online at http://npg.nature.com/ reprintsandpermissions/

How to cite this article: Zhou, L. et al. Tau association with synaptic vesicles cause presynaptic dysfunction. Nat. Commun. 8, 15295 doi: 10.1038/ncomms15295 (2017).

Publisher's note: Springer Nature remains neutral with regard to jurisdictional claims in published maps and institutional affiliations.

This work is licensed under a Creative Commons Attribution 4.0 International License. The images or other third party material in this article are included in the article's Creative Commons license, unless indicated otherwise in the credit line; if the material is not included under the Creative Commons license, users will need to obtain permission from the license holder to reproduce the material. To view a copy of this license, visit http://creativecommons.org/licenses/by/4.0/

(C) The Author(s) 2017 